Research Article

\title{
Grasping Torque Optimization for a Dexterous Robotic Hand Using the Linearization of Constraints
}

\author{
Peng Jia $\mathbb{D}^{1},{ }^{1}$ Lei Wu $\mathbb{D},{ }^{1}$ Gang Wang, ${ }^{1}$ Wei Na Geng $\mathbb{D},{ }^{2}$ Feihong Yun $\mathbb{D}^{1},{ }^{1}$ and Ning Zhang ${ }^{1}$ \\ ${ }^{1}$ College of Mechanical and Electrical Engineering, Harbin Engineering University, Harbin 150001, Heilongjiang Province, China \\ ${ }^{2}$ Heilongjiang Institute of Technology, Harbin 150001, Heilongjiang Province, China \\ Correspondence should be addressed to Peng Jia; 13633605161@139.com
}

Received 21 July 2019; Revised 14 October 2019; Accepted 30 October 2019; Published 23 November 2019

Academic Editor: Ioannis Kostavelis

Copyright (C) 2019 Peng Jia et al. This is an open access article distributed under the Creative Commons Attribution License, which permits unrestricted use, distribution, and reproduction in any medium, provided the original work is properly cited.

\begin{abstract}
A new algorithm is proposed for the optimization of grasping torques. Previous work revealed that the optimization of the contact force can be simplified as a linear programming problem by replacing the nonlinear friction cone with a polyhedral cone. On this basis, further simplification and linearization is completed for various grasp contact constraints of the grasping system, and an optimization model is established with the minimum sum-of-squares of joint torques as an objective function. A timely and effective algorithm based on key constraint sets is then established. This methodology optimizes the joint torque directly and facilitates the control of the dexterous hand. A numerical example and experimental results show that the optimization algorithm has high precision and good real-time performance. The joint torque output obtained using the proposed algorithm was $27.6 \%$ lower than the result of the previous algorithm. The optimization algorithm can thus be used for optimal grasping control of a dexterous hand.
\end{abstract}

\section{Introduction}

In the action of grasping, the mechanical dexterous hand needs to control the joint torque according to the condition of the external wrench so that the contact forces counterbalance the external wrench and meet the friction constraint at each place of contact. However, subject to the same external wrench, a contact force that conforms to the stable grasp and friction constraints is not unique, and the torque at each joint is also not unique. Owing to the limitations of the structure and driving ability of the dexterous hand joint, the maximum driving torque output by each joint is limited. To make the best use of the driving torque of each joint, the torque of each joint needs to be reasonably assigned, and this problem comes down to the optimization of the grasping torque in the joint torque space of the dexterous hand. There are presently two methods of obtaining the optimal joint torque.

(1) The contact force is optimized according to a certain contact force index in the contact force space, and the contact force is then mapped to the joint torque space to obtain the optimal joint torque

Kerr and Roth [1] proposed an early method of optimizing the grasping force, which solved the optimal force using a polyhedron to linearize the friction cone and involved linear programming. Cheng et al. $[2,3]$ converted the friction constraint into a set of linear inequalities by replacing the nonlinear friction cone with a polyhedral cone and simplified the contact force optimization as a linear programming problem. Afterward, Klein et al. $[4,5]$ put forward the simplex algorithm and the interior-point algorithm based on linear programming to solve the optimization problem. Zhang and Gruver [6] decomposed the contact force space into subspaces and developed a method of determining the connectivity dimension of the subspace relative to the object that was being grasped, thus simplifying the analysis of the contact force distribution. Zhen et al. [7] recently developed an optimization algorithm to calculate a set of initial and minimum forces more quickly than was possible in earlier calculations. In addition, Zhu et al. [8-13] 
proposed a variety of linear optimization methods for the contact force to reduce the amount of calculation; these methods were studied in terms of simplification of the pointcontact friction cone, contact force optimization index, linear programming method, and real-time performance. While linear programming methods may be advantageous in terms of the computational efficiency, they may provide grasping forces that are too conservative or violate nonlinear friction constraints. The accuracy of the linear programming method also depends in part on how many segments are used in the linear approximation of the friction constraint. Adding more segments can improve the solution optimality but at the cost of computational efficiency, and the calculation time becomes great even for a moderate number of segments.

From the perspective of nonlinear programming, Buss et al. [14] verified that adopting the friction cone constraint is equivalent to testing the positive definiteness of symmetric matrices, and the grasping force optimization is expressed as a convex optimization problem on a Riemannian manifold with linear constraints. On this basis, algorithms have been further explored and developed. Buss et al. [15] proposed a general-class Dikin-type algorithm for strictly convex quadratic continuously cost differentiable functions, guaranteeing a unique solution to the semidefinite programming problem related to the optimization of a smart grasping force. Helmke et al. [16] put forward a new compact representation of the friction cone constraint, reducing the complexity of the optimization problem. Remond et al. $[17,18]$ proposed a method that is based on the minimization cost function and gave an analytical solution; however, the solution does not ensure that friction constraints are met. Liu et al. [19] reported how to simplify the structure of the constraints and select appropriate initial conditions, step size, and stopping criteria. Gazeau et al. [20] turned the problem into a minimum distance problem and solved it using a gradient projection method. Cornella et al. [21] gave a similar expression but solved the dual form rather than the primal form. Lippiello et al. $[22,23]$ proposed a solution to lower the computational complexity of the problem by dynamically reducing the number of effective torque constraints, and they imported the previous one-handed operation into the two-handed operating system. The aforementioned various algorithms do not linearize the friction cone; the force optimization is thus more accurate, and the results obtained using the nonlinear method are more reasonable.

However, such nonlinear gradient algorithms also have the disadvantage of requiring a valid initial value of the grasping force before starting the iteration. To address this disadvantage, Han et al. [24] proposed a MAX-DET algorithm that uses linear matrix inequalities for initial grasping; however, the algorithm encounters singularity problems in some cases. Boyd and Wegbreit [25] constructed a simple single-valued optimization problem to get the initial value. Employing a Lagrangian multiplier, Wang et al. [26] proposed an iterative method of obtaining the initial values that meet the friction constraint by adjusting the weighting factor of the normal force. Chen et al. [27] gave a linear combination for the initial value of the grasping force. Wang et al. [28] recently proposed an iterative method for the initial value of the grasping force and safe edge detection, ensuring that the initial safe value meets the friction cone constraint in the optimization process of the gradient algorithm.

To reduce the computational complexity, Zheng et al. $[29,30]$ proposed a now-popular method that divides the algorithm into online and offline phases. By solving as many offline problems as possible, suboptimal but still acceptable solutions can be found with reduced online computing time. Using sparse matrix techniques and reducing matrix dimensions, Li [31] and Ji and Peng [32] divided the calculations of a solution into online and offline components. In addition, nonlinear methods were studied for this type of method because they have higher precision and higher computational speed than linear programming methods, which benefits online application. Wang et al. [33-35] proposed a primal-dual interior-point algorithm based on the kernel function to solve this convex quadratic programming over circular cones. Chen et al. [36] proposed a method implemented by using the fmincon function based on sequential quadratic programming in MATLAB optimization toolbox.

As more joints are used in modeling a system, the procedures required to derive the relevant motion equations become more laborious and complicated. Hollerbach [37] developed Lagrangian formulation of manipulator dynamics and verified that recursive formulations based on the Lagrangian or Newton-Euler dynamics offer the best method of dynamics calculation. Korayem et al. [38, 39] presented new methods to derive the dynamic equations of manipulators using the recursive Gibbs-Appell formulation while using hierarchical optimal control for path planning of mobile manipulator. Agarwal et al. [40] investigated the dynamic behaviour of serial chains with degrees of freedom and used a Recursive Dynamic Simulator which is based on the Newton-Euler formulation and the decoupled natural orthogonal complement matrices to simulate the dynamics of these systems. Recently, Korayem et al. [41-43] proposed a path planning method for mobile manipulator, which decouples the whole system into a group of subsystems and performs hierarchical optimal control, and they directly put forward additional constraints in the task space of mobile manipulators to resolve extra degrees of freedom introduced by the base mobility.

The most appropriate method for real-time grasping and control is to use neural networks. In recent years, deep learning has shown promising results. Xia et al. [44] developed a neural network algorithm that converges to the optimal solution at any initial point. Other methods have been developed for general problems similar to the grasping force optimization problem [45-47]. Levine et al. [48] described a learning-based approach to hand-eye coordination for robotic grasping from monocular images, a large convolutional neural network was trained to predict the probability that task space motion of the gripper will result in successful grasps, and then used this network to servo the gripper in real time to achieve successful grasps. Qin et al. 
[49] used convolution neural network to find the optimal configuration of grasping stability prediction by tactile data, which improves the success rate of grasping. Chao et al. [50] proposed a deep learning-based object detection method for a five-fingered industrial robot hand model, which improves the accuracy of robotic grasp in some uncertain environments. To optimize the grasping force of the grasping manipulation, Ko et al. [51, 52] proposed a semismooth Newton gripping force optimization method, which converts friction constraints into Cartesian products of secondorder cones.

(2) Optimize the joint torque in the joint torque space according to a certain joint torque optimization index.

Li et al. [53] used the maximization of the carrying capacity of the dexterous hand joint as the objective function and used an iterative algorithm to optimize the nonlinear joint torque. However, the nonlinearity of the constraints and the complexity of the objective function introduce difficulties to the optimization process in terms of convergence and real-time processing. And, little work on this type of method has been carried out.

Both linear programming and nonlinear programming are carried out in the contact force space. The dimension of the optimization problem increases with the number of contact points and the complexity of the contact constraint, which will increase the amount of calculation. In addition, the joint torque mapped from the optimal contact force may not be the best in the joint space. At the same time, the nonlinearity of constraints brings difficulties to the convergence and real time of the optimization process in the joint torque space.

The present paper proposes a new efficient algorithm for obtaining the optimal grasping torque of a joint of the dexterous hand. On the basis of previous studies, this paper further simplifies and linearizes various grasping contact constraints of the grasping system and establishes an optimization model with the minimum sum-of-squares of joint torques as an objective function. Then, a timely and effective algorithm based on key constraint sets is established. Unlike the majority of algorithms that first obtain the optimal contact force and then convert the force into the joint torque to realize control of the dexterous hand, this algorithm obtains the optimal grasping torque directly, simplifying the control process and reducing the control time. Compared with the result obtained using the algorithm proposed by $\mathrm{Li}$ et al. [53], the joint torque obtained by our algorithm is weaker and the optimization result is more reasonable.

Grasping experiments were carried out on a variety of different shapes for each grasping system. Comparing the optimized joint torque output with the original output, the effectiveness of the grasping torque optimization method based on the key constraint set is verified.

The remainder of the paper is structured as follows. Section 2 is devoted to the linearization of the contact constraint, Section 3 presents the simplification of grasping constraints, Section 4 develops the mathematical optimization model and algorithm, Section 5 presents numerical results, Section 6 presents experiments, and Section 7 presents conclusions and proposes future work.

\section{Linearization of the Contact Constrain}

This section presents basic knowledge on grasp contact constraints. Various grasp contact constraints are linearized by replacing the nonlinear single friction cone, paired friction cones, and friction ellipsoid with the linear single polyhedral cone, paired polyhedral cones, and ellipsoidal polyhedron, respectively.

2.1. Contact Constraint. The dexterous hand grasps and manipulates an object through contact. There are three types of contact between the finger and object, as depicted in Figure 1: (a) frictionless point contact (FPC), where the contact force comprises only the normal force $f_{z}$; (b) point contact with friction (PCwF), where the contact force comprises the normal force component $f_{z}$ and tangential force components $f_{x}$ and $f_{y}$; and (c) soft-finger contact (SFC), where the contact force comprises the normal force component $f_{z}$, tangential force components $f_{x}$ and $f_{y}$, and normal torque $m_{z}$. SFC can be divided into two types: SFC with a linear approximation (SFCl) and SFC with an elliptical approximation (SFCe), with the difference between SFCl and SFCe being that the contact force needs to meet different contact constraints [7, 54-58].

To avoid slippage and separation at the point of contact, for the above three contact types, the contact force must satisfy the following contact constraints:

$$
\begin{aligned}
& \text { FPC }: f_{i z}>0, \\
& \text { PCwF }: \sqrt{f_{i x}^{2}+f_{i y}^{2}} \leq \mu_{1} f_{i z} ; \quad f_{i z}>0, \\
& \text { SFCl }: \frac{\sqrt{f_{i x}^{2}+f_{i y}^{2}}}{u_{1}}+\frac{\left|m_{i z}\right|}{u_{2}} \leq f_{i z} ; \quad f_{i z}>0, \\
& \text { SFCe }: \sqrt{\frac{\left(f_{i x}^{2}+f_{i y}^{2}\right)}{u_{1}^{2}}+\frac{m_{i z}^{2}}{u_{2}^{\prime 2}} \leq f_{i z} ; \quad f_{i z}>0 .}
\end{aligned}
$$

Here, $f_{i z}$ is the normal force component of the contact, $f_{i x}$ and $f_{i y}$ are the tangential force components, $m_{i z}$ is the normal torque, $u_{1}$ is the tangential friction coefficient, and $u_{2}$ and $u_{2}^{\prime}$ are, respectively, the torsional friction coefficients for $\mathrm{SFCl}$ and SFCe.

2.2. Linearization of the $P C w F$ Constraint. If the contact between the dexterous hand and object being grasped can be simplified as PCwF, the normal force component $f_{i z}$ and tangential force components $f_{i x}$ and $f_{i y}$ of each point of contact need to meet the nonlinear constraints determined by equation (2) during the grasping. The following discusses how to linearize the nonlinear constraint and simplify the contact constraint. 


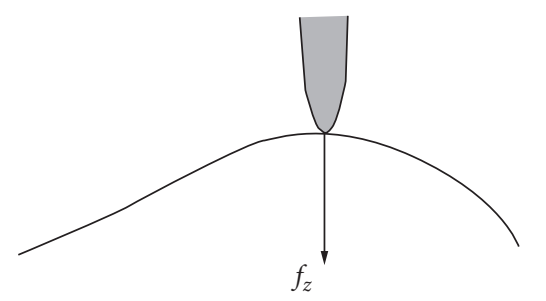

(a)

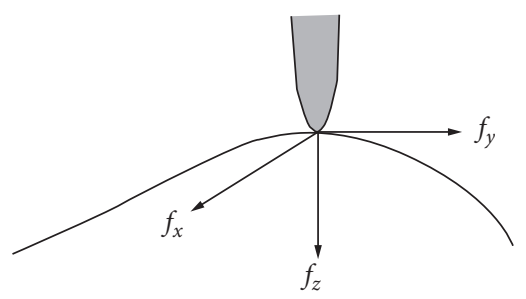

(b)

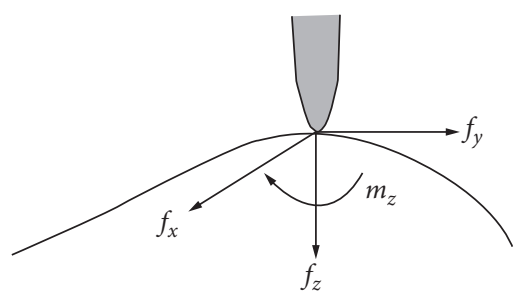

(c)

Figure 1: Three common contact types. (a) FPC. (b) PCwF. (c) SFC.

The contact force $\mathbf{f}_{i}=\left[\begin{array}{lll}f_{i x} & f_{i y} & f_{i z}\end{array}\right]^{T}$ inside the friction cone meets the condition of the PCwF constraint, as shown in Figure 2. Making an internal polyhedral cone that linearly simulates the friction cone, the contact force $\mathbf{f}_{i}$ located in the polyhedral cone will meet the condition of the PCwF constraint.

The faces of the polyhedral cone satisfy a linear equation, and the points inside the polyhedral cone meet a linear inequality equation. The linear polyhedral cone can then replace the friction cone and realize the linearization of the PCwF constraint. The linear inequality equation of the contact force that satisfies the polyhedral cone constraint is derived as follows.

Assuming that the internal polygon has $N$ sides, the vectors of the polygon cone sides can be expressed as

$$
\mathbf{S}_{j}=\left[\begin{array}{lll}
S_{j}^{1} & S_{j}^{2} & S_{j}^{3}
\end{array}\right]^{T}=\left[\begin{array}{lll}
\mu_{1} \cos \frac{2 \pi}{N} j & \mu_{1} \cos \frac{2 \pi}{N} j & 1
\end{array}\right]^{T},
$$

where $j=0,1,2, \ldots, N-1, N$.

The plane of the contact force formed by sides $S_{j}$ and $S_{j+1}$ can be expressed as

$$
\left[\begin{array}{lll}
a_{j} & b_{j} & c_{j}
\end{array}\right]\left[\begin{array}{l}
f_{x} \\
f_{y} \\
f_{z}
\end{array}\right]=0,
$$

where $\quad a_{j}=S_{j}^{2} S_{j+1}^{3}-S_{j}^{3} S_{j+1}^{2}, b_{j}=S_{j}^{3} S_{j+1}^{1}-S_{j+1}^{3} S_{j}^{1}$, and $c_{j}=$ $S_{j}^{1} S_{j+1}^{2}-S_{j+1}^{1} S_{j}^{2}, j=0,1,2, \ldots, N-1, N$.

The contact force $\mathbf{f}_{i}$ inside the polyhedral cone can, therefore, be expressed using a linear inequality:

$$
\left[\begin{array}{ccc}
a_{0} & b_{0} & c_{0} \\
a_{1} & b_{1} & c_{1} \\
\vdots & \vdots & \vdots \\
a_{N-1} & b_{N-1} & c_{N-1} \\
0 & 0 & 1
\end{array}\right]_{(N+1) \times 3} \quad\left[\begin{array}{l}
f_{i x} \\
f_{i y} \\
f_{i z}
\end{array}\right] \geq 0 .
$$

Because the polyhedral cone is inscribed in the friction cone, the contact force satisfying equation (7) must satisfy the condition of the $\mathrm{PCwF}$ constraint determined by equation (2). Meanwhile, the friction constraint is simplified to the linear inequality equation. It is noted that the contact force inside the interlayer part between the polyhedral cone and friction cone also satisfies the condition of the PCwF constraint. However, this part of the feasible contact force is eliminated in the linearization process, and the contact force

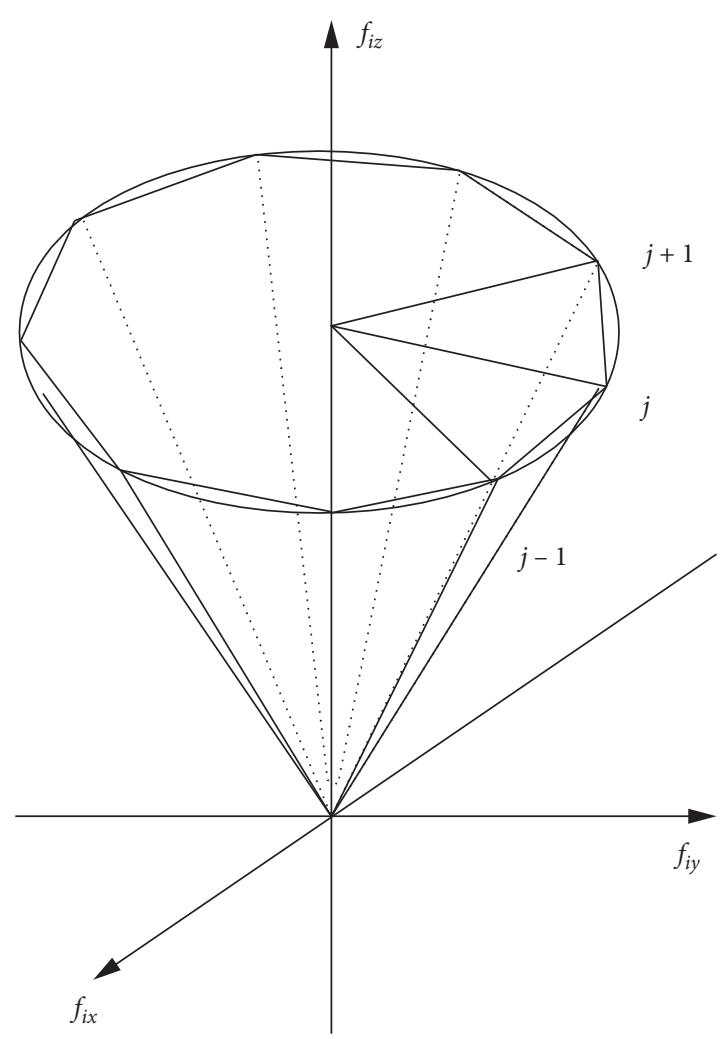

FIGURE 2: Linearization of the PCwF constraint.

$\mathbf{f}_{i}$ determined by the linearization constraint is, therefore, also relatively conservative.

The above analysis reveals that increasing the inscribed polyhedron side number $N$ results in higher accuracy of the linearization, and a less conservative contact force provides more linearized inequalities, leading to a larger computation and worse real-time performance. The situation is just the opposite when reducing the number of sides $N$ of the inscribed polyhedron. The number of inscribed polyhedron sides should thus be reasonably selected according to the accuracy and real-time processing requirements in the linearization process.

2.3. Linearization of the SFCl Constraint. To simplify the $\mathrm{SFCl}$ constraint, the nonlinear inequality constraint given as equation (3) can be written equivalently as 


$$
\sqrt{f_{i x}^{2}+f_{i y}^{2}} \leq u_{1} f_{i z}-\frac{\left|m_{i z}\right| u_{1}}{u_{2}} ; \quad f_{i z}>0
$$

In the Cartesian coordinate frame $o-f_{x} f_{y} m_{z}$, as shown in Figure 3, the contact force $\mathbf{f}_{i}=\left[\begin{array}{llll}f_{i x} & f_{i y} & f_{i z} & m_{i z}\end{array}\right]^{T}$ satisfying the above constraint is distributed in the double cone. The position of the vertices and the radius of the round base of the double cone are related to the normal force $f_{i z}$ at the point of contact, the coordinates of the upper and lower vertices are, respectively, ( $\left(\begin{array}{lll}0 & 0 & \mu_{2} f_{i z}\end{array}\right)$ and $\left(\begin{array}{lll}0 & 0 & -\mu_{2} f_{i z}\end{array}\right)$, and the radius of the round base is $\mu_{1} f_{i z}$.

An internal bipyramid is taken to simulate the double cone, as shown in Figure 3. Because the faces of the bipyramid satisfy a linear equation, a bipyramid is used in place of the friction double cone to linearize the $\mathrm{SFCl}$ constraint. The linear inequality of the contact force that satisfies the bipyramid constraints is derived as follows.

Assuming that the inscribed polygon in the round base of the double cone has $N$ sides, the coordinates of the inscribed polygon points in the coordinate frame $o-f_{x} f_{y} m_{z}$ can be expressed as

$$
f^{j}=\left(\begin{array}{lll}
f_{x}^{j} & f_{y}^{j} & f_{z}^{j}
\end{array}\right)=\left(\mu_{1} f_{i z} \cos \frac{2 \pi}{N} j \mu_{1} f_{i z} \sin \frac{2 \pi}{N} j \quad 0\right),
$$

where $j=0,1,2, \ldots, N-1, N$.

The upper and lower vertices of the bipyramid and vertices of the $\mathrm{N}$-gon in the round base form $2 \mathrm{~N}$ planes. From the coordinates of the bipyramid vertices and equation (9), the linear inequality equation that satisfies the contact constraint of the bipyramid is obtained as

$$
\left\{\begin{array}{l}
\mu_{2}\left(s_{j}-s_{j+1}\right) f_{i x}+\mu_{2}\left(c_{j+1}-c_{j}\right) f_{i y}+\mu_{1} \mu_{2} k f_{i z}-\mu_{1} k m_{i z} \geq 0, \\
\mu_{2}\left(s_{j}-s_{j+1}\right) f_{i x}+\mu_{2}\left(c_{j+1}-c_{j}\right) f_{i y}+\mu_{1} \mu_{2} k f_{i z}+\mu_{1} k m_{i z} \geq 0,
\end{array}\right.
$$

where $s_{j}=\sin (2 \pi j / N), c_{j}=\cos (2 \pi j / N), k=\sin (2 \pi j / N)$, and $j=0,1,2, \ldots, N-1$.

Because the normal contact force $f_{i z}>0$, the linear inequality constraint of the SFCl can be expressed as

$$
\left[\begin{array}{cccc}
a_{0} & b_{0} & l_{1} & l_{2} \\
a_{1} & b_{1} & l_{1} & l_{2} \\
\vdots & \vdots & \vdots & \vdots \\
a_{N-1} & b_{N-1} & l_{1} & l_{2} \\
a_{0} & b_{0} & l_{1} & -l_{2} \\
\vdots & \vdots & \vdots & \vdots \\
a_{N-1} & b_{N-1} & l_{1} & -l_{2} \\
0 & 0 & 1 & 0
\end{array}\right]_{(2 N+1) \times 4} \quad\left[\begin{array}{c}
f_{i x} \\
f_{i y} \\
f_{i z} \\
m_{i z}
\end{array}\right] \geq 0
$$

where $a_{j}=\mu_{2}\left(s_{j}-s_{j+1}\right), b_{j}=\mu_{2}\left(c_{j+1}-c_{j}\right), l_{1}=\mu_{1} \mu_{2} k, l_{2}=$ $-\mu_{1} k, s_{j}=\sin (2 \pi j / N), c_{j}=\cos (2 \pi j / N), k=\sin (2 \pi j / N)$, and $j=0,1,2, \ldots, N-1$.



FIGURE 3: Linearization of the SFCl constraint.

2.4. Linearization of the SFCe Constraint. To linearize the SFCe constraint, the nonlinear inequality constraint equation (4) can be written equivalently as

$$
\frac{f_{i x}^{2}}{u_{1}^{2}}+\frac{f_{i y}^{2}}{u_{1}^{2}}+\frac{m_{i z}^{2}}{u_{2}^{\prime 2}} \leq f_{i z}^{2} ; \quad f_{i z}>0
$$

In the Cartesian coordinate frame $o-f_{x} f_{y} m_{z}$, as shown in Figure 4, the contact force $\mathbf{f}_{i}=\left[\begin{array}{cccc}f_{i x} & f_{i y} & f_{i z} & m_{i z}\end{array}\right]^{T}$ satisfying the above constraint is distributed in an ellipsoid. The radius of the circle formed by the ellipsoid and $f_{x} f_{y}$ coordinate plane and the vertexes of the ellipsoid are related to the normal force $f_{i z}$ at the point of contact. The radius of the circle is $\mu_{1} f_{i z}$ while the coordinates of the upper and lower vertices are, respectively, $\left(\begin{array}{lll}0 & 0 & u_{2}^{\prime} f_{i z}\end{array}\right)$ and $\left(\begin{array}{lll}0 & 0 & -u_{2}^{\prime} f_{i z}\end{array}\right)$.

The above analysis reveals that the contact force in the ellipsoid satisfies the condition of the SFCe constraint so that the linearization of the SFCe constraint becomes the linearization of the ellipsoid. As shown in Figure 4, the internal ellipsoidal polyhedron is used to linearly simulate the ellipsoid and linearize the contact constraint. The linear inequality equation of the contact force that satisfies the constraints of the ellipsoidal polyhedron is derived as follows.

The entire ellipsoid surface is marked with lines of latitude and longitude. The ellipsoid surface is uniformly distributed with $N$ longitude lines, and the longitude line that intersects the positive axis $f_{i x}$ of the ellipsoid coordinate frame is referred to as the zero-longitude line, which coincides with the Nth longitude line. The circle formed by the surface of the ellipsoid and the coordinate plane $f_{i x} f_{i y}$ of the 


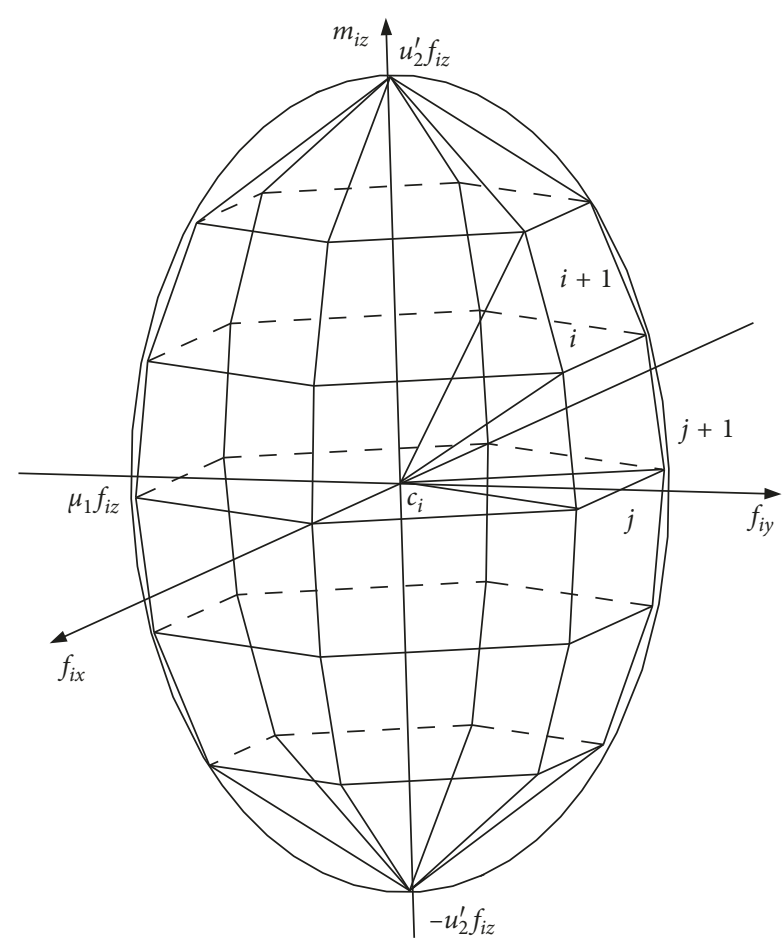

FIgURE 4: Linearization of the SFCe constraint.

ellipsoid coordinate frame is referred to as the zero-latitude line. The surfaces of the north and south semiellipsoids are equally divided by $K$ latitude lines, and the $K$ th latitude lines of the north and south semiellipsoids become the north and south vertices.

The upper semiellipsoid is selected as the research object; the coordinates of the north vertex are $\left(\begin{array}{llll}0 & 0 & u_{2}^{\prime} & f_{i z}\end{array}\right)$. The number of intersections of the latitude and longitude on the surface of the upper semiellipsoid is $n=K \times N$, and the coordinates of each point of intersection can be expressed as

$$
\left(f_{x}(i, j) f_{y}(i, j) m_{z}(i)\right)=\left(f_{z} l_{i} c_{j} f_{z} l_{i} s_{j} f_{z} l_{i} k_{i}\right)
$$

where $l_{i}=\mu_{1} \mu_{2}^{\prime} / \sqrt{\mu_{1}^{2} k_{i}^{2}+\mu_{2}^{\prime 2}}, k_{i}=\tan (\pi i / 2 K), s_{j}=\sin (2 \pi j /$ $N), s_{j}=\sin (2 \pi j / N), c_{j}=\cos (2 \pi j / N), j=0,1,2, \ldots, N-1$, and $i=0,1,2, \ldots, K-1$.

These $K \times N+1$ points are combined into an ellipsoidal polyhedron with $N \times K$ planes. The surface equation of the upper semiellipsoidal polyhedron can be described as

$$
\begin{aligned}
& \left(l_{i} k_{i}-l_{i+1} k_{i+1}\right)\left(s_{j}-s_{j+1}\right) f_{i x}+\left(l_{i} k_{i}-l_{i+1} k_{i+1}\right)\left(c_{j+1}-c_{j}\right) f_{i y} \\
& \quad-l_{i} l_{i+1} k\left(k_{i+1}-k_{i}\right) f_{i z}+k\left(l_{i}-l_{i+1}\right) m_{i z}=0
\end{aligned}
$$

where $j=0,1,2, \ldots, N-1, i=0,1,2, \ldots, K-1$, and $k=$ $\sin (2 \pi / N)$.

The surface equation of the lower semiellipsoidal polyhedron can be obtained from symmetry as

$$
\begin{aligned}
& \left(l_{i} k_{i}-l_{i+1} k_{i+1}\right)\left(s_{j}-s_{j+1}\right) f_{i x} \\
& \quad+\left(l_{i} k_{i}-l_{i+1} k_{i+1}\right)\left(c_{j+1}-c_{j}\right) f_{i y}-l_{i} l_{i+1} k\left(k_{i+1}-k_{i}\right) f_{i z} \\
& \quad-k\left(l_{i}-l_{i+1}\right) m_{i z}=0
\end{aligned}
$$

where $j=0,1,2, \ldots, N-1, i=0,1,2, \ldots, K-1$, and $k=$ $\sin (2 \pi / N)$.

Combining the above $2 N \times K$ surface linear equations, the linear equation of the contact force that satisfies the constraint of the ellipsoidal polyhedron is obtained as

$$
\begin{aligned}
& \left(l_{i} k_{i}-l_{i+1} k_{i+1}\right)\left(s_{j+1}-s_{j}\right) f_{i x} \\
& \quad+\left(l_{i} k_{i}-l_{i+1} k_{i+1}\right)\left(c_{j}-c_{j+1}\right) f_{i y} \\
& \quad-l_{i} l_{i+1} k\left(k_{i}-k_{i+1}\right) f_{i z} \pm k\left(l_{i+1}-l_{i}\right) m_{i z} \geq 0,
\end{aligned}
$$

where $j=0,1,2, \ldots, N-1, i=0,1,2, \ldots, K-1$, and $k=$ $\sin (2 \pi / N)$.

Because the normal contact force $f_{i z}>0$, the linear inequality constraint of the SFCe can be expressed as

$$
\left[\begin{array}{cccc}
a_{00} & b_{00} & c_{0} & d_{0} \\
\vdots & \vdots & \vdots & \vdots \\
a_{(N-1) 0} & b_{(N-1) 0} & c_{0} & d_{0} \\
\vdots & \vdots & \vdots & \vdots \\
a_{0(K-1)} & b_{0(K-1)} & c_{K-1} & d_{K-1} \\
\vdots & \vdots & \vdots & \vdots \\
a_{(N-1)(K-1)} & b_{(N-1)(K-1)} & c_{K-1} & d_{(K-1)} \\
a_{00} & b_{00} & c_{0} & -d_{0} \\
\vdots & \vdots & \vdots & \vdots \\
a_{(N-1) 0} & b_{(N-1) 0} & c_{0} & -d_{0} \\
\vdots & \vdots & \vdots & \vdots \\
a_{0(K-1)} & b_{0(K-1)} & c_{K-1} & -d_{K-1} \\
\vdots & \vdots & \vdots & \vdots \\
a_{(N-1)(K-1)} & b_{(N-1)(K-1)} & c_{K-1} & -d_{(K-1)} \\
0 & 0 & 1 & 0
\end{array}\right]_{(2 N K+1) \times 4} \quad\left[\begin{array}{c}
f_{i x} \\
f_{i y} \\
f_{i z} \\
m_{i z}
\end{array}\right] \geq 0,
$$

where $a_{j i}=\left(l_{i} k_{i}-l_{i+1} k_{i+1}\right)\left(s_{j+1}-s_{j}\right), b_{j i}=\left(l_{i} k_{i}-l_{i+1} k_{i+1}\right)$ $\left(c_{j}-c_{j+1}\right), c_{i}=-l_{i} l_{i+1} k\left(k_{i}-k_{i+1}\right), d_{i}=k\left(l_{i+1}-l_{i}\right), j=0,1$, $2, \ldots, N-1, \quad i=0,1,2, \ldots, K-1, \quad k=\sin (2 \pi / N), \quad l_{i}=$ $\mu_{1} \mu_{2}^{\prime} / \sqrt{\mu_{1}^{2} k_{i}^{2}+\mu_{2}^{\prime 2}}, k_{i}=\tan (\pi i / 2 K), s_{j}=\sin (2 \pi j / N)$, and $c_{j}=$ $\cos (2 \pi j / N)$.

\section{Simplification of Constraints}

This section expresses the contact force using the joint torque and external wrench. The equilibrium constraint and friction constraint that need to be satisfied in grasping are simplified as a linear constraint with the joint torque as the variable, while an optimization model is established with 
linear constraints as optimization constraints and with the minimum sum-of-squares of joint torques as the objective function. The solution of the optimization model then comes down to a quadratic function optimization problem under linear constraints, and a timely and effective algorithm for the optimal grasping torque based on key constraint sets is proposed.

3.1. Decomposition of the Contact Force. In grasping, a multifinger dexterous hand must meet the equilibrium conditions

$$
\begin{aligned}
\mathbf{G} \mathbf{f}=\mathbf{w} \\
\mathbf{J}^{T} \mathbf{f}=\boldsymbol{\tau}
\end{aligned}
$$

Here, $\mathbf{G} \in \mathbf{R}^{6 \times n}$ is the grasping matrix, $\mathbf{f} \in \mathbf{R}^{n}$ is the contact force applied at points of contact on the fingers, $\mathbf{w} \in \mathbf{R}^{6}$ is the external wrench applied to the object, $\mathbf{J} \in \mathbf{R}^{n \times m}$ is the Jacobian matrix of the dexterous hand, and $\tau \in \mathbf{R}^{m}$ is the joint torque of a finger of the dexterous hand. Detailed static and kinematic equations that need to be satisfied in the grasping process and general solutions of the equations are given in the literature $[59,60]$.

The contact force $\mathbf{f}$ is an $N$-dimensional vector space, which can be decomposed into four mutually orthogonal subspaces:

$$
\begin{aligned}
\boldsymbol{\Gamma}_{h 2} & =\left\{\mathbf{f}_{h 2} \mid \mathbf{G f}_{h 2}=0, \mathbf{J}^{T} \mathbf{f}_{h 2}=0\right\}, \\
\boldsymbol{\Gamma}_{h 1} & =\left\{\mathbf{f}_{h 1} \mid \mathbf{G f}_{h 1}=0, \mathbf{J}^{T} \mathbf{f}_{h 1}=\boldsymbol{\tau}, \quad \boldsymbol{\tau} \neq 0\right\}, \\
\boldsymbol{\Gamma}_{p 2} & =\left\{\mathbf{f}_{p 2} \mid \mathbf{G} \mathbf{f}_{p 2}=\mathbf{w}, \mathbf{J}^{T} \mathbf{f}_{p 2}=0, \quad \mathbf{w} \neq 0\right\}, \\
\boldsymbol{\Gamma}_{p 1} & =\left\{\mathbf{f}_{p 2} \mid \mathbf{G f}_{p 2}=\mathbf{w}, \mathbf{J}^{T} \mathbf{f}_{p 1}=\boldsymbol{\tau}, \quad \mathbf{w} \neq 0, \boldsymbol{\tau} \neq 0\right\},
\end{aligned}
$$

where $\mathbf{f}_{h 2}$ can be called a passive internal force because it cannot be provided by joint torque $\tau$ and is unable to equilibrate the external wrench $\mathbf{w}$. There is no passive internal force $\mathbf{f}_{h 2}$ for the rigid grasp, where both objects and fingers are considered as rigid bodies, but the passive internal force $\mathbf{f}_{h 2}$ acts as a preload for the elastic grasp where both objects and fingers are considered elastomers. Mainly used to adjust and control the contact friction, $\mathbf{f}_{h 1}$ can be called an active internal force because it can be provided by joint torque $\tau$, but it is unable to equilibrate the external wrench $\mathbf{w}$. Equilibrating the external wrench $\mathbf{w}$ through the mechanical structure and friction passively, $\mathbf{f}_{p 2}$, which cannot be provided by joint torque $\tau$, can be called a passive external force. $\mathbf{f}_{p 1}$ can be called an active external force because it can be provided by joint torque $\tau$ and equilibrate the external wrench $\mathbf{w}$.

The contact force $\mathbf{f}$ can therefore be decomposed as

$$
\mathbf{f}=\mathbf{f}_{h 2}+\mathbf{f}_{h 1}+\mathbf{f}_{p 2}+\mathbf{f}_{p 1},
$$

where $\mathbf{f}_{h 2}$ is the passive internal force, $\mathbf{f}_{h 1}$ is the active internal force, $\mathbf{f}_{p 2}$ is the passive external force, and $\mathbf{f}_{p 1}$ is the active external force.
The mapping relationship of the external wrench $\mathbf{w}$, contact force $\mathbf{f}$, and joint torque $\boldsymbol{\tau}$ is shown in Figure 5, where $R\left(\mathbf{J}^{T}\right)$ is the range for $\mathbf{J}^{T}$ and $R(\mathbf{G})$ is the range for $\mathbf{G}$.

The representation of the contact force $\mathbf{f}$ is directly related to the research problem. In order to simplify the contact force, the method of undetermined coefficient is used to express the contact force $\mathbf{f}$.

Because $\mathbf{G f}_{h 2}=0$ and $\mathbf{J}^{T} \mathbf{f}_{h 2}=0, \mathbf{f}_{h 2}$ belonging to the null space of matrix $\mathbf{A}=\left[\begin{array}{c}\mathbf{G} \\ \mathbf{J}^{T}\end{array}\right]$ can be expressed as

$$
\mathbf{f}_{h 2}=\mathbf{N}_{A} \mathbf{x}_{A}, \quad \mathbf{x}_{A} \in R^{n-\operatorname{rank}(A)} .
$$

Because $\mathbf{G f}_{h 1}=0$ and $\mathbf{N}_{A}^{T} \mathbf{f}_{h 1}=0, \mathbf{f}_{h 1}$ belonging to the null space of matrix $\mathbf{B}=\left[\begin{array}{c}G \\ \mathbf{N}_{A}^{T}\end{array}\right]$ can be expressed as

$$
\mathbf{f}_{h 1}=\mathbf{N}_{B} \mathbf{x}_{B}, \quad \mathbf{x}_{B} \in R^{n-\operatorname{rank}(B)} .
$$

Because $\mathbf{J}^{T} \mathbf{f}_{p 2}=0$ and $\mathbf{N}_{A}^{T} \mathbf{f}_{p 2}=0, \mathbf{f}_{p 2}$ belonging to the null space of matrix $\mathbf{C}=\left[\begin{array}{c}\mathbf{J}^{T} \\ \mathbf{N}_{A}^{T}\end{array}\right]$ can be expressed as

$$
\mathbf{f}_{p 2}=\mathbf{N}_{C} \mathbf{x}_{C}, \quad \mathbf{x}_{C} \in R^{n-\operatorname{rank}(C)} .
$$

Because $\mathbf{N}_{C}^{T} \mathbf{f}_{p 1}=0, \mathbf{N}_{B}^{T} \mathbf{f}_{p 1}=0$, and $\mathbf{N}_{A}^{T} \mathbf{f}_{p 1}=0, \mathbf{f}_{p 1}$ belonging to the null space of matrix $\mathbf{D}=\left[\begin{array}{l}\mathbf{N}_{C}^{T} \\ \mathbf{N}_{B}^{T} \\ \mathbf{N}_{A}^{T}\end{array}\right]$ can be
expressed as

$$
\mathbf{f}_{p 1}=\mathbf{N}_{D} \mathbf{x}_{D}, \quad \mathbf{x}_{D} \in R^{n-\operatorname{rank}(D)}
$$

The contact force $\mathbf{f}$ of the dexterous hand can be obtained using the method of the undetermined coefficient as

$$
\mathbf{f}=\mathbf{N}_{A} \mathbf{x}_{A}+\mathbf{N}_{B} \mathbf{x}_{B}+\mathbf{N}_{C} \mathbf{x}_{C}+\mathbf{N}_{D} \mathbf{x}_{D}
$$

To further simplify the constraint of the grasping force on the basis of the linearization of the contact constraint, the contact force $\mathbf{f}$ is decomposed into the form of a joint torque $\boldsymbol{\tau}$ and external wrench $\mathbf{w}$.

Equations (18) and (19) can be combined to give

$$
\text { Bf }=\mathbf{d} \text {, }
$$

where $\mathbf{d}=\left[\begin{array}{c}\mathbf{w} \\ \boldsymbol{\tau}\end{array}\right]$ and $\mathbf{B}=\left[\begin{array}{c}\mathbf{G} \\ \mathbf{J}^{T}\end{array}\right]$

Substituting equation (26), $\mathbf{G f}_{h 2}=0$, and $\mathbf{J}^{T} \mathbf{f}_{h 2}=0$ into equation (27), we have

$$
\mathbf{Q} \mathbf{x}=\mathbf{d},
$$

where $\mathbf{Q}=\left[\begin{array}{lll}\mathbf{B N}_{D} & \mathbf{B N}_{C} & \mathbf{B N}_{B}\end{array}\right]$ and $\mathbf{x}=\left[\begin{array}{lll}\mathbf{x}_{D} & \mathbf{x}_{C} & \mathbf{x}_{B}\end{array}\right]^{T}$.

The solution obtained by solving the above matrix equation is

$$
\mathbf{x}=\left(\mathbf{Q}^{T} \mathbf{Q}\right)^{-1} \mathbf{Q}^{T} \mathbf{d}
$$

Substituting the above solution into equation (26), we have 




Figure 5: Decomposition of the contact force space.

$$
\mathbf{f}=\mathbf{N}_{A} \mathbf{x}_{A}+\mathbf{Z}_{1} \mathbf{w}+\mathbf{Z}_{2} \tau .
$$

The dexterous-hand grasping system is generally rigid, allowing the effect of the first item to be ignored. Equation (30) can then be simplified as

$$
\mathbf{f}=\mathbf{Z}_{1} \mathbf{w}+\mathbf{Z}_{2} \boldsymbol{\tau},
$$

where $\mathbf{Z}_{1}$ is the matrix of external wrench equivalent coefficients and $\mathbf{Z}_{2}$ is the matrix of joint torque equivalent coefficients; both are obtained from $\mathbf{G}$ and $\mathbf{J}$ and reflect the shape of the grasp and object being grasped.

3.2. Conversion of Variables. To optimize the grasping force of the dexterous hand in the joint torque space, the constraint of the contact force must be converted into the constraint of the joint torque. The contact force has previously been broken down into the joint torque and external wrench. Substituting the contact force into the linearized contact constraint, the linear inequality constraint with the joint torque as the variable can be obtained.

The unified form of the linearization formula of each contact constraint mentioned above has not been obtained.

For the PCwF constraint, equation (7) can be transformed as

$$
\begin{aligned}
\mathbf{S}_{i} \mathbf{f}_{i} \geq & 0, \\
\mathbf{S}_{i} & =\left[\begin{array}{ccc}
a_{0} & b_{0} & c_{0} \\
a_{1} & b_{1} & c_{1} \\
\vdots & \vdots & \vdots \\
a_{N-1} & b_{N-1} & c_{N-1} \\
0 & 0 & 1
\end{array}\right]_{(N+1) \times 3} \\
\mathbf{f}_{i} & =\left[\begin{array}{c}
f_{i x} \\
f_{i y} \\
f_{i z}
\end{array}\right] .
\end{aligned}
$$

Assuming that the dexterous hand has $n$ points of contact with the object and all contacts are of type PCwF, the total contact force $\mathbf{f}$ can be expressed as $\mathbf{f}=\left[\begin{array}{llll}\mathbf{f}_{1}^{T} & \mathbf{f}_{2}^{T} & \cdots & \mathbf{f}_{n}^{T}\end{array}\right]^{T}$, where $\mathbf{f}_{i}=\left[\begin{array}{lll}f_{i x} & f_{i y} & f_{i z}\end{array}\right]^{T}$.
Let $\mathbf{S}=-$ Blockdiag $\left(\mathbf{S}_{i}\right)$. The contact constraint inequalities for the $n$ points of contact of the dexterous hand can then be expressed as

$$
\mathbf{S}_{(n N+n) \times(3 n)} \mathbf{f}_{3 n \times 1} \geq 0 .
$$

For the SFCl constraint, equation (11) can be rewritten as

$$
\begin{aligned}
\mathbf{S}_{i} \mathbf{f}_{i} \geq & 0, \\
\mathbf{S}_{i} & =\left[\begin{array}{cccc}
a_{0} & b_{0} & l_{1} & l_{2} \\
a_{1} & b_{1} & l_{1} & l_{2} \\
\vdots & \vdots & \vdots & \vdots \\
a_{N} & b_{N} & l_{1} & l_{2} \\
a_{0} & b_{0} & l_{1} & -l_{2} \\
\vdots & \vdots & \vdots & \vdots \\
a_{N} & b_{N} & l_{1} & -l_{2} \\
0 & 0 & 1 & 0
\end{array}\right]_{(2 N+1) \times 4} \\
\mathbf{f}_{i} & =\left[\begin{array}{c}
f_{i x} \\
f_{i y} \\
f_{i z} \\
m_{i z}
\end{array}\right] .
\end{aligned}
$$

Assuming that the dexterous hand has $n$ points of contact with the object and all contacts are of type SFCl, the total contact force $\mathbf{f}$ can be expressed as $\mathbf{f}=\left[\begin{array}{llll}\mathbf{f}_{1}^{T} & \mathbf{f}_{2}^{T} & \cdots & \mathbf{f}_{n}^{T}\end{array}\right]^{T}$, where $\mathbf{f}_{i}=\left[\begin{array}{llll}f_{i x} & f_{i y} & f_{i z} & m_{i z}\end{array}\right]^{T}$.

Let $\mathbf{S}=\operatorname{Blockdiag}\left(\mathbf{S}_{i}\right)$. The contact constraint inequalities for the $n$ points of contact of the dexterous hand can then be expressed as

$$
\mathbf{S}_{(2 N n+n) \times(4 n)} \mathbf{f}_{4 n \times 1} \geq 0 .
$$

For the SFCe constraint, equation (17) can be rewritten as

$$
\begin{gathered}
\mathbf{S}_{i} \mathbf{f}_{i} \geq 0, \\
\mathbf{S}_{i}=\left[\begin{array}{cccc}
a_{00} & b_{00} & c_{0} & d_{0} \\
\vdots & \vdots & \vdots & \vdots \\
a_{(N-1) 0} & b_{(N-1) 0} & c_{0} & d_{0} \\
\vdots & \vdots & \vdots & \vdots \\
a_{0(K-1)} & b_{0(K-1)} & c_{K-1} & d_{K-1} \\
\vdots & \vdots & \vdots & \vdots \\
a_{(N-1)(K-1)} & b_{(N-1)(K-1)} & c_{K-1} & d_{(K-1)} \\
a_{00} & b_{00} & c_{0} & -d_{0} \\
\vdots & \vdots & \vdots & \vdots \\
a_{(N-1) 0} & b_{(N-1) 0} & c_{0} & -d_{0} \\
\vdots & \vdots & \vdots & \vdots \\
a_{0(K-1)} & b_{0(K-1)} & c_{K-1} & -d_{K-1} \\
\vdots & \vdots & \vdots & \vdots \\
a_{(N-1)(K-1)} & b_{(N-1)(K-1)} & c_{K-1} & -d_{(K-1)} \\
0 & 0 & 1 & 0
\end{array}\right]_{(2 N K+1) \times 4},
\end{gathered},
$$$$
\left[\begin{array}{l}
f_{i x} \\
f_{i y} \\
f_{i z} \\
m_{i z}
\end{array}\right] \geq 0 .
$$ 
Assuming that the dexterous hand has $n$ points of contact with the object and all contacts are of type SFCe, the total contact force $\mathbf{f}$ can be expressed as $\mathbf{f}=\left[\begin{array}{llll}\mathbf{f}_{1}^{T} & \mathbf{f}_{2}^{T} & \cdots & \mathbf{f}_{n}^{T}\end{array}\right]^{T}$, where $\mathbf{f}_{i}=\left[\begin{array}{llll}f_{i x} & f_{i y} & f_{i z} & m_{i z}\end{array}\right]^{T}$.

Let $\mathbf{S}=$ Blockdiag $\left(\mathbf{S}_{i}\right)$. The contact constraint inequalities for the $n$ points of contact of the dexterous hand can then be expressed as

$$
\mathbf{S}_{(2 N k n+n) \times(4 n)} \mathbf{f}_{4 n \times 1} \geq 0 .
$$

The unified form of equations (33), (35), and (37) is

$$
\text { Sf } \geq 0 \text {. }
$$

Substituting equation (31) into equation (38), the linear inequality constraint with joint torque as the variable that applies to different friction constraints can be expressed as

$$
\mathrm{SZ}_{2} \tau+\mathrm{SZ}_{1} \mathbf{w} \geq 0
$$

Here, the matrix of external wrench equivalent coefficients $\mathbf{Z}_{1}$ and the matrix of joint torque equivalent coefficients $\mathbf{Z}_{2}$ are related to the situation of grasping and the shape of the object, and the matrix $\mathbf{S}$ is related to the contact type and the linearization accuracy of the frictional contact. When the dexterous hand grasps the object stably in a certain grasping situation, to satisfy the force equilibrium and the contact force constraint, the joint torque and external wrench must satisfy the linear constraint of equation (39).

\section{Mathematical Model and Algorithm}

This section establishes the optimization model with linear constraints as optimization constraints and with the minimum sum-of-squares of joint torques as the objective function. A real-time and effective algorithm of the active constraint method based on key constraint sets is then established.

4.1. Mathematical Optimization Model. When the grasping situation and contact are determined, to grasp the object stably, the dexterous hand must meet the following constraints:

(1) The contact force equilibrates the external wrench

(2) The contact force is inside the friction cone

(3) The joint torque equilibrates the contact force

The contact force is passively generated when the finger is in contact with the object, and the joint torque is directly controllable. Subject to the same external wrench, there are various joint torque distribution schemes that satisfy the constraint. To optimize the grasping force in the joint torque space and obtain the optimal joint torque, it is necessary to construct a reasonable joint torque objective function. Considering the limitation of the power output and structural size of the dexterous hand driver, the maximum output torque of the joint is limited. It is always expected that the greatest external wrench be equilibrated with the weakest possible driving torque, and the objective function of joint torque optimization can thus be expressed as

$$
\sigma=\sum_{i=1}^{m}\left(\tau_{i}\right)^{2}=\boldsymbol{\tau}^{T} \boldsymbol{\tau},
$$

where $\boldsymbol{\tau}_{i}$ is the torque of the dexterous hand joint and $m$ is the number of dexterous hand joints.

Taking the minimum sum-of-squares of joint torques as the objective function, the joint torque as the optimization variable, and the equilibrium constraint that satisfies the joint torque and the friction constraint of the contact force as the optimization constraints, the mathematical model of the optimization of the grasping torque for the dexterous hand in the joint torque space can be described as

$$
\begin{aligned}
& \text { Min } \sigma=\sum_{i=1}^{m}\left(\tau_{i}\right)^{2}=\tau^{T} \tau \\
& \text { subject to (2), (3), (4), (18), and (19). }
\end{aligned}
$$

Because the optimization constraint is nonlinear with the joint torque as the variable, the optimization problem comes down to a nonlinear programming problem with equality and inequality constraints. Although there are corresponding calculation methods in mathematics, they usually require many iterative operations and do not meet the requirement of real-time processing. It is thus necessary to simplify the optimization constraints appropriately to meet the needs of the real-time control of dexterous hands. The linear constraint with the joint torque as the variable is then used instead of the nonlinear constraint, and the mathematical optimization model is simplified as

$$
\begin{array}{r}
\sigma=\sum_{i=1}^{m}\left(\tau_{i}\right)^{2}=\boldsymbol{\tau}^{T} \boldsymbol{\tau}, \\
\text { subject to } \quad \mathbf{S Z}_{2} \boldsymbol{\tau}+\mathbf{S Z}_{1} \mathbf{w} \geq 0 .
\end{array}
$$

All constraints of the simplified mathematical optimization model become linear inequality constraints, and the joint torques satisfying the constraints constitute a feasible convex set in the joint torque space. When using two joints for grasping, the physical meaning of equation (42) can be visually described as shown in Figure 6 . The shadow of the polygon represents the feasible set of joint torque $\tau$ that satisfies the constraints of equation (42), and the polygon is a convex polygon because all constraints are linear. Several circles represent the distribution of joint torques $\tau$ that obey the functional equation $\sigma=\left(\tau_{1}\right)^{2}+\left(\tau_{2}\right)^{2}$.

In Figure 6, circle 1 does not intersect with the feasible convex set and it is thus not possible to obtain the joint torque $\tau$ on the circle. Circle 3 has multiple intersections with the feasible convex set; the joint torque $\tau$ at the intersections satisfies all the constraints but does not provide the smallest $\sigma$, and an optimal solution is thus not obtained. Circle 2 has a single tangent point A with the feasible convex set; this is the optimum point satisfying all constraints and providing the smallest $\sigma$, and joint torques $\tau_{1}$ and $\tau_{2}$ corresponding to point $\mathrm{A}$ are thus optimal.

When equation (42) is generalized to multiple joint torque spaces, the torque $\tau$ satisfies all linear constraints is a convex set in the joint torque space, the objective function 




Figure 6: Optimal joint torque of grasping with two joints.

becomes a generalized sphere with a variable diameter, and the tangent point of the generalized sphere and convex set is the optimal solution. Because all the constraints are linear and the objective function is quadratic, the optimal solution problem becomes a quadratic programming problem. The algorithm for solving this quadratic programming is discussed as follows.

4.2. Algorithm. Equation (42) can be written equivalently as

$$
\text { Min } \quad \sigma=\sum_{i=1}^{m}\left(\tau_{i}\right)^{2}=\boldsymbol{\tau}^{T} \boldsymbol{\tau}
$$

subject to $\mathbf{H} \boldsymbol{\tau} \geq \mathbf{c}$,

where $\quad \boldsymbol{\tau}=\left[\begin{array}{llll}\tau_{1} & \tau_{2} & \cdots & \tau_{m}\end{array}\right]^{T} \in \mathbf{R}^{m}, \quad \mathbf{H}=\mathbf{S Z}_{2}, \quad$ and $\mathbf{c}=-\mathbf{S Z}_{1} \mathbf{w}$.

To solve the above problem, we select the initial feasible point of joint torque $\tau_{1}$ that satisfies the constraint, take the point of the joint torque as the initial point, remove the constraint set that is useless at the point of the joint torque in the force equilibrium and the linear friction constraint, and seek the key constraint set at the point of the joint torque. Under the simplified equality constraint, the Lagrange multiplier method is used to obtain the minimum value of the objective function, which is the optimal feasible point of the joint torque.

Then, with the better feasible point of the joint torque as the new initial point, the above process can be repeated for a limited time, and the optimal joint torque satisfying the above constraint can be obtained.

Assuming that the feasible point of joint torque $\tau_{k}$ is obtained in the $k$ th iteration and that the key constraint set that takes effect at the point of the joint torque is $\mathbf{I}_{k}$, the problem is simplified as the quadratic function minimization problem under an equality constraint:

$$
\begin{aligned}
& \text { Min } \quad \sigma=\boldsymbol{\tau}^{T} \boldsymbol{\tau}, \\
& \text { subject to } \mathbf{h}_{i}^{T} \boldsymbol{\tau}=c_{i}, i \in \mathbf{I}_{k},
\end{aligned}
$$

where $\mathbf{h}_{i}$ is an $m$-dimensional vector, comprising elements of the $i$ th column of the constraint matrix $\mathbf{H}^{T}$.
The Lagrange multiplier method is used to solve the quadratic function minimization problem under the above equality constraints. The optimal joint torque is denoted as $\boldsymbol{\tau}_{k}^{\prime}$, and it is assumed that $\mathbf{d}_{k}=\boldsymbol{\tau}_{k}^{\prime}-\boldsymbol{\tau}_{k}$. The following steps are carried out depending on the situation:

(1) If $\boldsymbol{\tau}_{k}+\mathbf{d}_{k}$ still satisfies the constraints while $\mathbf{d}_{k} \neq 0$, then in the next iteration, the point of joint torque $\boldsymbol{\tau}_{k+1}=\boldsymbol{\tau}_{k}+\mathbf{d}_{k}$ is taken as the initial point.

(2) If $\boldsymbol{\tau}_{k}+\mathbf{d}_{k}$ no longer satisfies the constraints, then in the next iteration, the initial joint torque is $\boldsymbol{\tau}_{k+1}=\boldsymbol{\tau}_{k}+\lambda_{k} \mathbf{d}_{k}$. The following question is how to choose a suitable $\lambda_{k}$ such that $\boldsymbol{\tau}_{k+1}$ for any $i$, where $i \notin \mathbf{I}_{k}$, meets the constraint

$$
\mathbf{h}_{i}^{T}\left(\boldsymbol{\tau}_{k}+\lambda_{k} \mathbf{d}_{k}\right) \geq \mathbf{c}_{i}
$$

When $\mathbf{h}_{i}^{T} \mathbf{d}_{k} \geq 0$ and if the step size $\lambda_{k} \geq 0$, equation (45) can always be established. When $\mathbf{h}_{i}^{T} \mathbf{d}_{k}<0$, to establish equation (45), the step size $\lambda_{k}$ needs to meet the condition

$$
\lambda_{k} \leq \min \left\{\frac{\mathbf{c}_{i}-\mathbf{h}_{i}^{T} \boldsymbol{\tau}_{k}}{\mathbf{h}_{i}^{T} \mathbf{d}_{k}} \mid i \notin \mathbf{I}_{k}, \quad \mathbf{h}_{i}^{T} \mathbf{d}_{k}<0\right\} .
$$

To get a better point of torque, take $\lambda_{k}=\min \left\{1, \lambda_{k}^{\prime}\right\}$, where $\lambda_{k}^{\prime}$ is the rightmost term of equation (46). If there is an index $p$ that is not in the key constraint set but satisfies

$$
\lambda_{k}=\frac{\mathbf{c}_{p}-\mathbf{h}_{p}^{T} \boldsymbol{\tau}_{k}}{\mathbf{h}_{p}^{T} \mathbf{d}_{k}}<1,
$$

then $\mathbf{h}_{p}^{T} \mathbf{d} \geq c_{p}$ is also a key constraint at the feasible point of torque $\tau_{k+1}$. The constraint corresponding to the index $p$ is added to the key constraint set $\mathbf{I}_{k+1}$. The process returns to the beginning of the question, and a Lagrange multiplier is used to obtain the optimal solution of equation (44) again.

(3) If $\mathbf{d}_{k}=0$, then the optimal solution of equation (44) is $\boldsymbol{\tau}_{k}$, but the point of torque $\boldsymbol{\tau}_{k}$ may not be the optimal solution of equation (43). The Lagrange multiplier $v_{i}^{(k)}$ is further calculated under all key constraints to determine whether the point of torque $\tau_{k}$ is the optimal solution of equation (43).

If $\forall i \in \mathbf{I}_{k}$ satisfy $v_{i}^{(k)} \geq 0$, then the point of torque $\boldsymbol{\tau}_{k}$ is the optimal solution of equation (45), and the whole problem is solved.

If $\exists q \in \mathbf{I}_{k}$ for which $v_{q}^{(k)}<0$, then the point of torque $\tau_{k}$ is not the optimal solution and can thus be optimized in some direction. Therefore, let $v_{r}^{(k)}=\min \left\{v_{r}^{(k)} \mid i \in \mathbf{I}_{k}\right\}$ and the $r$ th constraint equation is removed from the key constraint set $I_{k}$, the process returns to the beginning of the question, and a Lagrangian multiplier is used to obtain the optimal solution of equation (44) again.

The specific algorithm is given as follows:

Step 1: Select the initial data. Give the initial feasible point of joint torque $\boldsymbol{\tau}_{1}$. The corresponding key constraint set is $\mathbf{I}_{1}$. Let $k=1$. 
Step 2: Solve the equality constraint problem. Solve equation (44) and set the optimal solution as $\boldsymbol{\tau}_{k}^{\prime}$. Assume that $\mathbf{d}_{k}=\boldsymbol{\tau}_{k}^{\prime}-\boldsymbol{\tau}_{k}$.

If $\mathbf{d}_{k}=0$, then go to Step 4 .

Otherwise, let $\lambda_{k}=\min \left\{1, \lambda_{k}^{\prime}\right\}, \boldsymbol{\tau}_{k+1}=\boldsymbol{\tau}_{k}+\lambda_{k} \mathbf{d}_{k}$, and go to Step 3.

Step 3: Modify the key constraint set.

If $\lambda_{k}<1$, then let $\mathbf{I}_{k+1}=\mathbf{I}_{k} \cup\{p\}, k=k+1$, and return to Step 2.

If $\lambda_{k}=1$, then let $k=k+1$, and go to Step 4 .

Step 4: Determine whether the point of the torque is the optimal solution and calculate the Lagrangian multiplier $v_{i}^{(k)}$ corresponding to all active constraints, where $i \in \mathbf{I}_{k}$, and mark it as $v_{r}^{(k)}=$ $\min \left\{v_{i}^{(k)} \mid i \in I_{k}\right\}$.

If $v_{r}^{(k)} \geq 0$, the iteration is terminated and the optimal solution is $\tau_{k}$.

Otherwise, delete index $r$ from $\mathbf{I}_{k}$ and return to Step 2. The flow of the above process is shown in Figure 7.

\section{Numerical Examples}

This section provides a specific example of the grasping torque simulation, verifies the accuracy and real-time performance of the grasping optimization method, and analyzes the effect of linearization accuracy on the optimization results.

In the grasping system shown in Figure 8, the contact model is SFCl, the rod lengths are $l_{1}=l_{3}=2$ and $l_{2}=l_{4}=1$, and the object is a flat disc with radius $R=1 . O-x y z$ is the object coordinate frame established at the center of the disc, and $c_{i}-x_{i} y_{i} z_{i}(i=1,2)$ denotes a contact coordinate frame established at a point of contact. Assume that the friction coefficients of the points of contact are $\mu_{1}=0.5$ and $\mu_{2}=0.2$. The contact force is expressed as $\mathbf{f}=\left[\begin{array}{llllllll}f_{x_{1}} & f_{y_{1}} & f_{z_{1}} & m_{z_{1}} & f_{x_{2}} & f_{y_{2}} & f_{z_{2}} & m_{z_{2}}\end{array}\right]^{T}$, the external wrench is expressed as $\mathbf{w}=\left[\begin{array}{llllll}F_{x} & F_{y} & F_{z} & M_{x} & M_{y} & M_{z}\end{array}\right]^{T}=$ $\left[\begin{array}{llllll}-1 & -1 & 0 & 0 & 0 & 1 / 2\end{array}\right]^{T}$, and the joint torque is expressed as $\boldsymbol{\tau}=\left[\begin{array}{llll}\tau_{1} & \tau_{2} & \tau_{3} & \tau_{4}\end{array}\right]^{T}$. The analysis of the grasping torque optimization results under different linearization precision is as follows. $\mathbf{J}^{T}$ are

The grasping matrix $\mathbf{G}$ and Jacobian transposed matrix

$$
\begin{aligned}
\mathbf{G} & =\left[\begin{array}{cccccccc}
1 & 0 & 0 & 0 & -1 & 0 & 0 & 0 \\
0 & -1 & 0 & 0 & 0 & -1 & 0 & 0 \\
0 & 0 & 1 & 0 & 0 & 0 & -1 & 0 \\
0 & 1 & 0 & 0 & 0 & -1 & 0 & 0 \\
1 & 0 & 0 & 0 & 1 & 0 & 0 & 0 \\
0 & 0 & 0 & 1 & 0 & 0 & 0 & -1
\end{array}\right], \\
\mathbf{J}^{T} & =\left[\begin{array}{cccccccc}
0 & -1 & 2 & 0 & 0 & 0 & 0 & 0 \\
0 & -1 & 0 & 0 & 0 & 0 & 0 & 0 \\
0 & 0 & 0 & 0 & 0 & 1 & -2 & 0 \\
0 & 0 & 0 & 0 & 0 & 1 & 0 & 0
\end{array}\right]
\end{aligned}
$$

The external wrench equivalent coefficient matrix $\mathbf{Z}_{1}$ and joint torque equivalent coefficient matrix $\mathbf{Z}_{2}$ are

$$
\mathbf{Z}_{1}=\left[\begin{array}{cccccc}
0.5 & 0 & 0 & 0 & 0.5 & 0 \\
0 & -0.3333 & 0.1 & 0.3 & 0 & 0 \\
0 & -0.1667 & 0.2 & 0.1 & 0 & 0 \\
0 & 0 & 0 & 0 & 0.5 & 0 \\
-0.5 & 0 & 0 & 0 & 0.5 & 0 \\
0 & -0.3333 & -0.1 & -0.3 & 0 & 0 \\
0 & -0.1667 & -02 & -0.1 & 0 & 0 \\
0 & 0 & 0 & 0 & 0 & -0.5
\end{array}\right],
$$

The contact is SFCl. To simplify the linearization of the contact constraint, $N$ is set to 4 , which means that the friction double cone is simplified into a four-sided double cone, and the linear inequality can be expressed as

$$
\begin{aligned}
\text { Blockdiag }\left(\mathbf{S}_{1}\right) \mathbf{f} \geq 0 \\
\mathbf{S}_{1}=\left[\begin{array}{cccc}
-0.2 & -0.2 & 0.1 & -0.5 \\
0.2 & -0.2 & 0.1 & -0.5 \\
0.2 & 0.2 & 0.1 & -0.5 \\
-0.2 & 0.2 & 0.1 & -0.5 \\
-0.2 & -0.2 & 0.1 & 0.5 \\
0.2 & -0.2 & 0.1 & 0.5 \\
0.2 & 0.2 & 0.1 & 0.5 \\
-0.2 & 0.2 & 0.1 & 0.5 \\
0 & 0 & 1 & 0
\end{array}\right] \text {. }
\end{aligned}
$$

Substituting the above results into equation (35), the optimization of constraints is obtained as

$$
\mathrm{H \tau} \geq \mathrm{c},
$$

where 


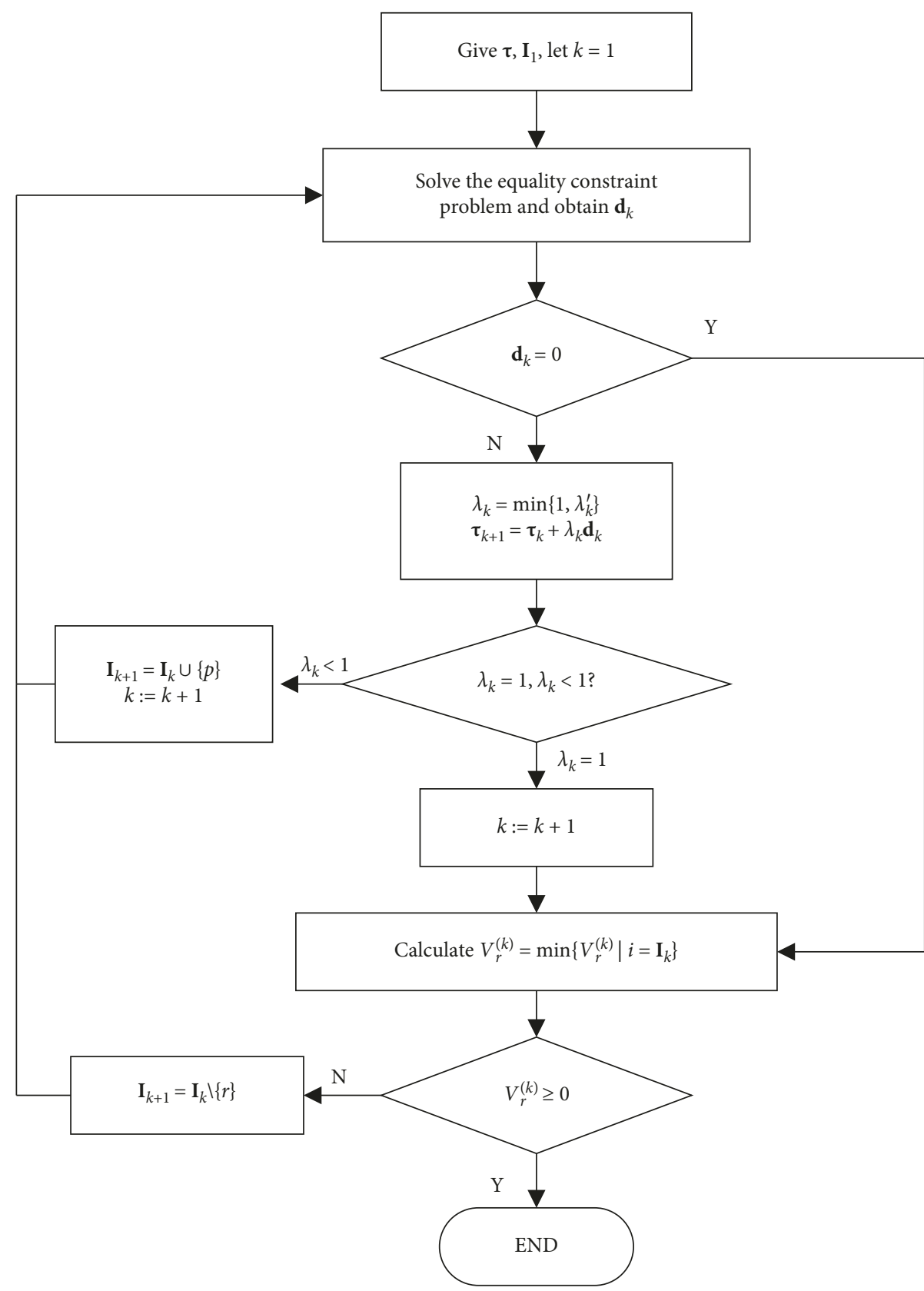

FIgURE 7: Optimization flow of the joint torque.

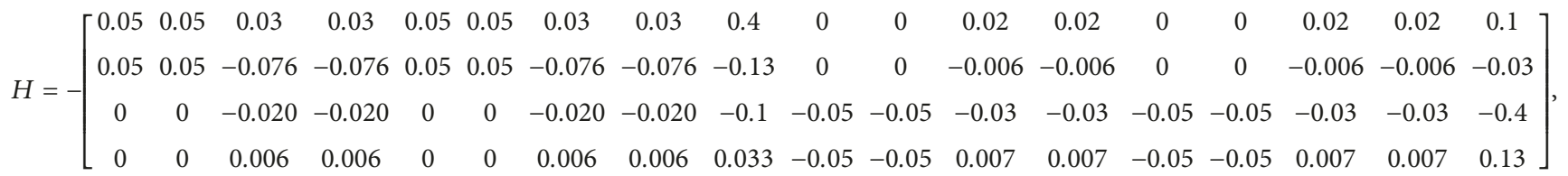

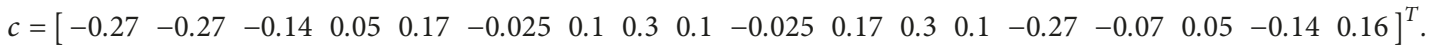

Initial values $\boldsymbol{\tau}_{1}=\left[\begin{array}{llll}7.5 & -2 & -7.5 & 2\end{array}\right]^{T}$ are selected and $I_{1}=(2,14)$. The joint torque is optimized using the optimization algorithm presented in this paper. The optimization result is $\tau_{\mathrm{opt}}=\left[\begin{array}{llll}4.5007 & 0.9993 & -4.5007 & -0.9993\end{array}\right]^{T}$ after eight iterations, and the optimal solution of the objective function is $\sigma=42.5105$. 


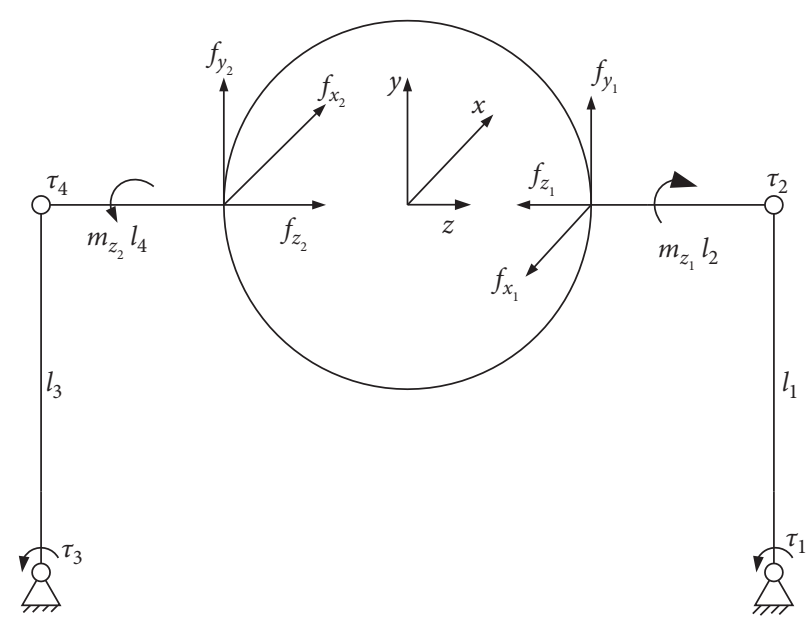

FIgURE 8: Optimization example of the joint torque.



FIgURE 9: Trends of the optimization result $\sigma$ and number of iterations $n$ with a change in $N$.

The optimization was conducted on a computer with a Pentium IV 2.8-GHz CPU and 1 GB RAM. The calculation time was less than $0.2 \mathrm{~s}$, and the real-time performance was thus good. The joint torque optimized using a method in the literature $[61]$ was $\tau_{\text {opt }}=\left[\begin{array}{llll}6.35 & 1.2 & -6.35 & -1.29\end{array}\right]^{T}$. A comparison of the optimized results reveals that the sum of the absolute value of joint torque obtained using the optimization method presented in the present paper is $27.6 \%$ smaller than that of the joint torque obtained using the method from the literature [61]. In addition, the joint torque obtained using the optimization method presented in the present paper is more reasonable.

The following analysis investigates the effect of linearization accuracy on the optimization results. Figure 9 shows the trends of the optimization result $\sigma$ and number of iterations $n$ as $N$ increases continuously, which means that the linearization error decreases, for optimization performed in the same way. It is seen that the optimization result $\sigma$ improves as $N$ increases, but the improvement in the linearization accuracy inevitably increases the linear inequality and the number of iterations in the optimization process, worsening the real-time performance.

\section{Experiment}

This section introduces the experimental system of the machine dexterous hand and carries out a grasping experiment on a variety of differently shaped objects.

6.1. Composition and Working Principle. Figure 10 shows that the experiment system of the dexterous hand mainly comprises the host computer control system, joint motion control system, dexterous hand mechanical body, fingertip force sensor processing system, and power source. As the host controller, the host computer mainly completes the grasping planning, the processing of fingertip force/torque information and human-computer interaction. The joint motion control system as the lower layer controller mainly performs the control of the force and positioning of the dexterous hand joint. The dexterous hand mechanical body acts as an actuator for grasping and manipulating the object. The fingertip force sensor processing system is primarily responsible for the measurement of the fingertip force/ torque. 


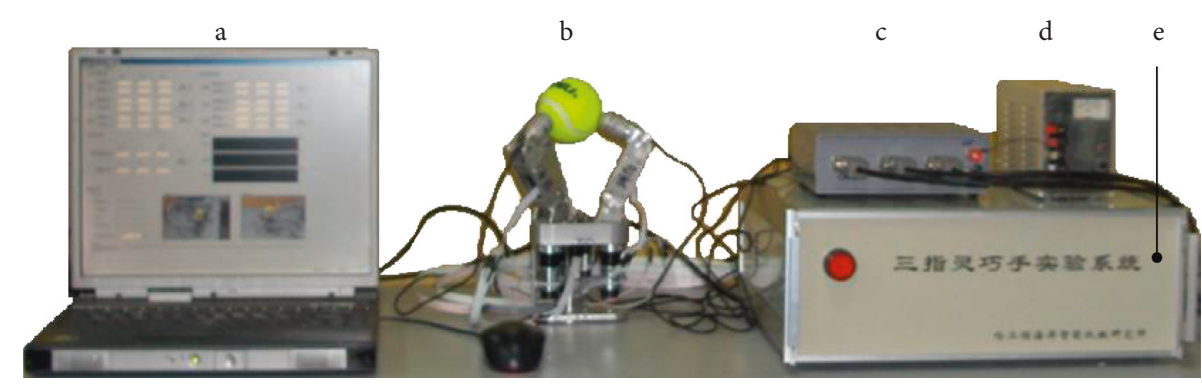

Figure 10: Experimental system of the dexterous hand. (a) Host computer control system. (b) Dexterous hand mechanical body. (c) Fingertip force sensor process. (d) Power. (e) Joint motion control system.

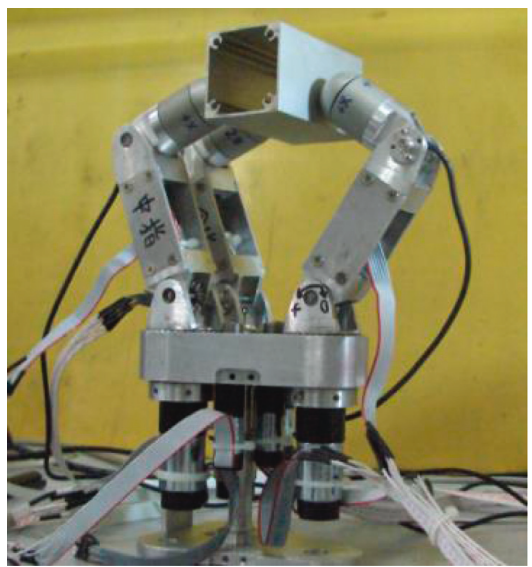

(a)

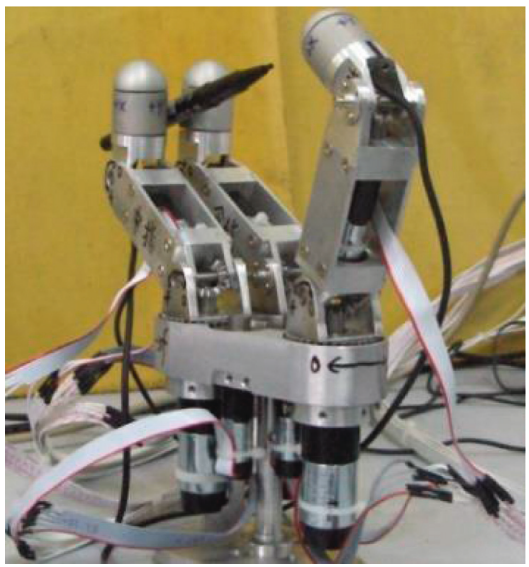

(d)

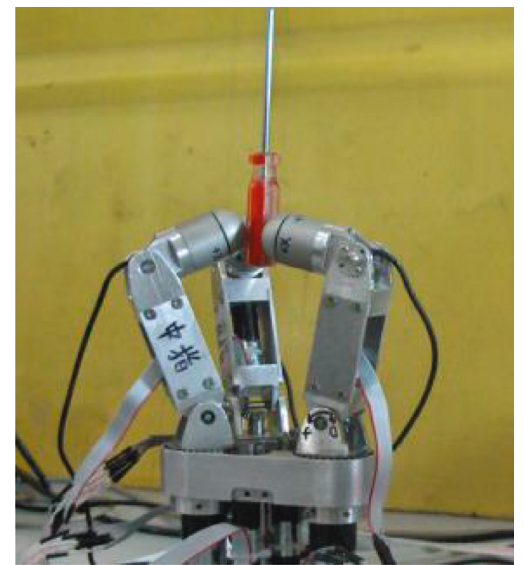

(b)

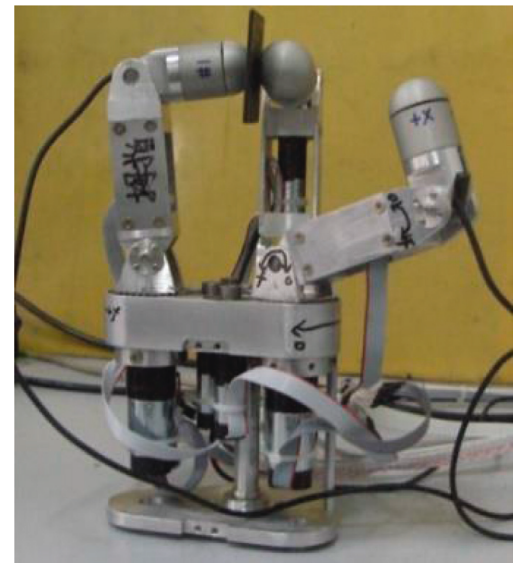

(e)

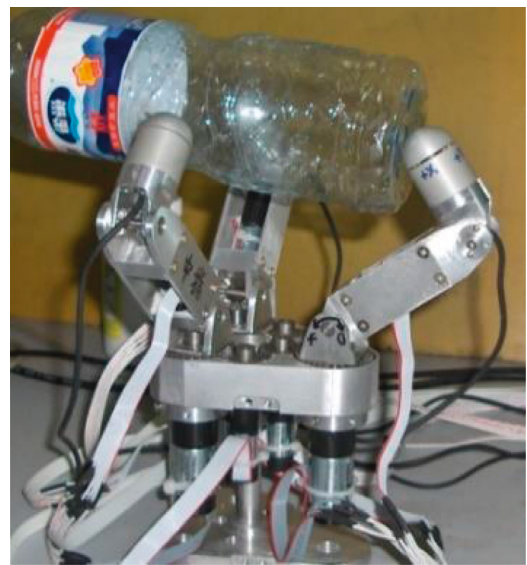

(c)

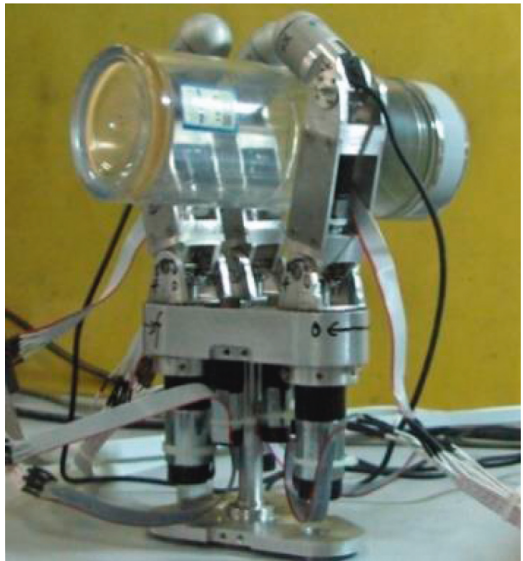

(f)

FIGURE 11: Optimization examination of grasping forces. (a) Grasping an aluminum block (cuboid). (b) Grasping a screwdriver (hexagonal prism). (c) Grasping a plastic bottle (cylinder). (d) Grasping a carbon pen (slender rod). (e) Grasping an iron piece (flat plate). (f) Grasping a cup vigorously (cylinder).

6.2. Optimization Experiment. Grasping experiments were carried out on a variety of object shapes, as shown in Figure 11. The algorithm module was first removed from the control program, and grasping experiments were then carried out for the differently shaped objects. After each hold stabilized, the host personal computer communicated with the joint servo control unit through the CAN bus, read and stored the torque information measured by each joint torque sensor, and obtained the output value of each joint torque of the dexterous hand when the optimization was not performed.

The algorithm module based on the key constraint set was then added to the control program and the above grasping experiment re-executed. Considering that the type of contact between the mechanical body of the dexterous hand and grasped object is $\mathrm{PCwF}$, to balance the precision and real-time processing of the grasping torque optimization, the contact constraint model was linearized into a 
TABLE 1: Absolute values of joint torque outputs before and after optimization $(\mathrm{N} \cdot \mathrm{m})$.

\begin{tabular}{|c|c|c|c|c|c|c|c|c|c|}
\hline & \multicolumn{3}{|c|}{ Thumb joint } & \multicolumn{3}{|c|}{ Forefinger joint } & \multicolumn{3}{|c|}{ Middle finger joint } \\
\hline & Bottom joint & Middle joint & Top joint & Bottom joint & Middle joint & Top joint & Bottom joint & Middle joint & Top joint \\
\hline \multicolumn{10}{|c|}{$\begin{array}{l}\text { Aluminum } \\
\text { block }\end{array}$} \\
\hline Before & 0.062 & 0.642 & 0.240 & 0.120 & 0.593 & 0.206 & 0.116 & 0.607 & 0.215 \\
\hline After & 0.048 & 0.509 & 0.190 & 0.106 & 0.587 & 0.166 & 0.110 & 0.590 & 0.171 \\
\hline \multicolumn{10}{|c|}{ Screwdriver } \\
\hline Before & 0.023 & 0.156 & 0.079 & 0.019 & 0.155 & 0.076 & 0.020 & 0.153 & 0.077 \\
\hline After & 0.021 & 0.106 & 0.065 & 0.020 & 0.096 & 0.065 & 0.019 & 0.098 & 0.065 \\
\hline \multicolumn{10}{|c|}{ Plastic bottle } \\
\hline Before & 0.052 & 0.342 & 0.157 & 0.155 & 0.453 & 0.160 & 0.161 & 0.463 & 0.161 \\
\hline After & 0.037 & 0.229 & 0.143 & 0.125 & 0.358 & 0.147 & 0.124 & 0.360 & 0.145 \\
\hline \multicolumn{10}{|c|}{ Carbon pen } \\
\hline Before & 0.230 & 0.102 & 0.104 & 0.212 & 0.102 & 0.097 & 0.008 & 0.030 & 0.006 \\
\hline After & 0.182 & 0.085 & 0.080 & 0.193 & 0.085 & 0.095 & 0.008 & 0.030 & 0.006 \\
\hline \multicolumn{10}{|l|}{ Iron piece } \\
\hline Before & 0.112 & 0.423 & 0.182 & 0.202 & 0.090 & 0.035 & 0.007 & 0.050 & 0.005 \\
\hline After & 0.105 & 0.328 & 0.150 & 0.175 & 0.085 & 0.033 & 0.007 & 0.050 & 0.004 \\
\hline \multicolumn{10}{|l|}{ Cup } \\
\hline Before & 0.162 & 0.524 & 0.140 & 0.150 & 0.413 & 0.136 & 0.146 & 0.397 & 0.135 \\
\hline After & 0.085 & 0.385 & 0.108 & 0.097 & 0.305 & 0.118 & 0.111 & 0.297 & 0.109 \\
\hline
\end{tabular}

linear polyhedral model with a face number $N=8$. The joint torque for a stable grasp before optimization was taken as the initial joint torque of the optimization algorithm. After several iterations, the joint torque was stably output and the host computer obtained the torque of each joint through the joint servo control unit again.

Outputs of each joint torque of the dexterous hand before and after optimization are given in Table 1 .

A comparison of the joint torque output of optimization with the original output shows that when the dexterous hand grasps the six differently shaped objects, the optimized joint torques are lower by varying degrees, especially in the case of the output torque of the middle finger joint.

\section{Conclusion and Future Work}

This paper presented an algorithm for obtaining the optimal grasping torque of a dexterous hand joint. Addressing the disadvantage of a previous algorithm that cannot achieve the driving torque of the joint directly, the optimization method presented in this paper optimizes the joint torque in the joint torque space. The proposed optimization model with linear optimization constraints replacing nonlinear optimization constraints reduces the large number of iterative computations. In addition, the optimization model is simplified using the minimum sumof-squares of joint torques as the objective function. Experiments show that the algorithm proposed in this paper can reduce the output torque of the dexterous hand joints, lighten the burden on the joint driver, improve the carrying capacity of the dexterous hand, and be used in the control system of a dexterous hand.

Despite the progress made in this paper and dexterous robotic hands shifting from prototypes to practical implementations, there remain many problems in grasping theory that need further solutions and in-depth exploration. Future work may include better indicators and more efficient optimization algorithms for grasping planning.

\section{Data Availability}

The data used to support the findings of this study are available from the corresponding author upon request.

\section{Conflicts of Interest}

The authors declare that they have no conflicts of interest.

\section{Acknowledgments}

This study was supported by the National Key Research and Development Program of China (2018YFC0310500) and the High-tech Ship Research Projects Sponsored by the Ministry of Industry and Information Technology (2018GXB01).

\section{References}

[1] J. Kerr and B. Roth, "Analysis of multifingered hands," The International Journal of Robotics Research, vol. 4, no. 4, pp. 3-17, 1986.

[2] F.-T. Cheng and D. E. Orin, "Efficient algorithm for optimal force distribution-the compact-dual LP method," IEEE Transactions on Robotics and Automation, vol. 6, no. 2, pp. 178-187, 1990.

[3] Y. H. Liu, "Qualitative test and force optimization of 3-D frictional form-closure grasps using linear programming," IEEE Transactions on Robotics \& Automation, vol. 15, no. 1, pp. 163-173, 1999.

[4] C. A. Klein and S. Kittivatcharapong, "Optimal force distribution for the legs of a walking machine with friction cone constraints," IEEE Transactions on Robotics and Automation, vol. 6, no. 1, pp. 73-85, 1990.

[5] Y. Zheng, C.-M. Chew, and A. H. Adiwahono, "A GJK-based approach to contact force feasibility and distribution for 
multi-contact robots," Robotics and Autonomous Systems, vol. 59, no. 3-4, pp. 194-207, 2011.

[6] Y. Zhang and W. A. Gruver, "Definition and force distribution of power grasps," in Proceedings of the IEEE international Conference on robotics and Automation, vol. 2, pp. 1373-1378, Nagoya, Japan, May 1995.

[7] Y. Zheng, M. C. Lin, and D. Manocha, "On computing reliable optimal grasping forces," IEEE Transactions on Robotics, vol. 28, no. 3, pp. 619-633, 2012.

[8] X. Y. Zhu and J. Wang, "Synthesis of force-closure grasps on 3-D objects based on the Q distance," IEEE Transactions on Robotics and Automation, vol. 19, no. 4, pp. 669-679, 2003.

[9] Y. Zheng and W.-H. Qian, "Limiting and minimizing the contact forces in multifingered grasping," Mechanism and Machine Theory, vol. 41, no. 10, pp. 1243-1257, 2006.

[10] T. Watanabe and T. Yoshikawa, "Grasping optimization using a required external force set," IEEE Transactions on Automation Science and Engineering, vol. 4, no. 1, pp. 52-66, 2007.

[11] R. Michalec and A. Micaelli, "Optimal tightening forces for multi-fingered robust manipulation," in Proceedings of the IEEE/RSJ International Conference on Intelligent Robots and Systems, pp. 4160-4167, St. Louis, MO, USA, October 2009.

[12] Y. Zheng and C. M. Chew, "A numerical solution to the rayshooting problem and its applications in robotic grasping," in Proceedings of the IEEE International Conference on Robotics and Automation, pp. 51-56, Kobe, Japan, May 2009.

[13] Y. Zheng, M. C. Lin, and D. Manocha, "A fast $n$-dimensional ray-shooting algorithm for grasping force optimization," in Proceedings of the IEEE International Conference on Robotics and Automation, pp. 1300-1305, Anchorage, AK, USA, May 2010.

[14] M. Buss, H. Hashimoto, and J. B. Moore, "Dextrous hand grasping force optimization," IEEE Transactions on Robotics and Automation, vol. 12, no. 3, pp. 406-418, 1996.

[15] M. Buss, L. Faybusovich, and J. B. Moore, "Dikin-type algorithms for dextrous grasping force optimization," The International Journal of Robotics Research, vol. 17, no. 8, pp. 831-839, 1998.

[16] U. Helmke, K. Huper, and J. B. Moore, "Quadratically convergent algorithms for optimal dextrous hand grasping," Ieee Transactions on Robotics and Automation, vol. 18, no. 2, pp. 138-146, 2002.

[17] C. Remond, V. Perdereau, and M. Drouin, "A multi-fingered hand control structure with on-line grasping force optimization," in Proceedings of the IEEE/ASME International Conference on Advanced Intelligent Mechatronics, vol. 2, pp. 804-809, Como, Italy, July 2001.

[18] J. P. Saut, C. Remond, V. Perdereau et al., "Online computation of grasping force in multi-fingered hands," IEEE/RSJ International Conference on Intelligent Robots and Systems, pp. 1223-1228, Edmonton, Canada, August 2005.

[19] G. Liu, J. Xu, and Z. Li, "On geometric algorithms for realtime grasping force optimization," IEEE Transactions on Control Systems Technology, vol. 12, no. 6, pp. 843-859, 2003.

[20] J. P. Gazeau, S. Zeghloul, and G. Ramirez, "Manipulation with a polyarticulated mechanical hand: a new efficient real-time method for computing fingertip forces for a global manipulation strategy," Robotica, vol. 23, no. 4, pp. 479-490, 2005.

[21] J. Cornellà, R. Suárez, R. Carloni, and C. Melchiorri, "Dual programming based approach for optimal grasping force distribution," Mechatronics, vol. 18, no. 7, pp. 348-356, 2008.

[22] V. Lippiello, B. Siciliano, and L. Villani, "Online dextroushand grasping force optimization with dynamic torque constraints selection," in Proceedings of the IEEE International
Conference on Robotics and Automation, pp. 2831-2836, Shanghai, China, May 2011.

[23] V. Lippiello, B. Siciliano, and L. Villani, "A grasping force optimization algorithm for multiarm robots with multifingered hands," IEEE Transactions on Robotics, vol. 29, no. 1, pp. 55-67, 2013.

[24] L. Han, J. C. Trinkle, and Z. X. Li, "Grasp analysis as linear matrix inequality problems," in Proceedings of the IEEE International Conference on Robotics and Automation, vol. 2, pp. 1261-1268, Detroit, MI, USA, May 1999.

[25] S. P. Boyd and B. Wegbreit, "Fast computation of optimal contact forces," IEEE Transactions on Robotics, vol. 23, no. 6, pp. 1117-1132, 2007.

[26] B. Wang, J. W. Li, and L. Hong, "Optimal grasping force computation for multi-fingered robot hand," Journal of Jilin University, vol. 38, no. 1, pp. 178-182, 2008.

[27] D. Chen, J. Li, and X. Wang, "Computation of multi-fingered grasping force with linear combination," Journal of Harbin Institute of Technology, vol. 45, no. 1, pp. 55-59, 2013.

[28] X. Wang, Y. Xiao, Y. Zhao et al., "Grasping force optimization algorithm of soft multi-fingered hand based on safety margin detection," ROBOT, vol. 39, no. 6, pp. 844-852, 2017.

[29] Y. Zheng and W.-H. Qian, "A fast procedure for optimizing dynamic force distribution in multifingered grasping," IEEE Transactions on Systems, Man and Cybernetics, Part B (Cybernetics), vol. 36, no. 6, pp. 1417-1422, 2006.

[30] B. R. Zuo and W. H. Qian, "A general dynamic force distribution algorithm for multifingered grasping," EEE Transactions on Systems, Man and Cybernetics, Part B (Cybernetics), vol. 30, no. 1, pp. 185-192, 2000.

[31] J. Li, "Real time force optimization algorithm of multi-fingered grasp," Chinese Journal of Mechanical Engineering, vol. 43, no. 12, pp. 144-149, 2007.

[32] T. Ji and X. Peng, "Task-based finger force analysis for robotics dexterous hand," in Proceedings of the 10th international Conference on computer science \& education, pp. 536-541, IEEE, Cambridge, UK, July 2015.

[33] Y. Bai, P. Ma, and J. Zhang, "A polynomial-time interiorpoint method for circular cone programming based on kernel functions," Journal of Industrial and Management Optimization, vol. 12, no. 2, pp. 739-756, 2015.

[34] G. Wang, X. Gao, and Y. Bai, "Primal-dual interior-point algorithms for convex quadratic circular cone optimization," Numerical Algebra, Control and Optimization, vol. 5, no. 2, pp. 211-231, 2015.

[35] Y.-Q. Bai, X.-R. Gao, and C.-J. Yu, “A primal-dual interiorpoint method for optimal grasping manipulation of multifingered hand-arm robots," Journal of the Operations Research Society of China, vol. 5, no. 1, pp. 177-192, 2017.

[36] J. Chen, D. Han, X. Wang et al., "Multi-fingered coordinated control for dexterous robotic hand," Journal of Mechanical Engineering, vol. 50, no. 5, p. 42, 2014.

[37] J. M. Hollerbach, "A recursive Lagrangian formulation of maniputator dynamics and a Comparative study of dynamics formulation complexity," IEEE Transactions on Systems, Man, and Cybernetics, vol. 10, no. 11, pp. 730-736, 1980.

[38] A. M. Shafei and M. H. Korayem, "Theoretical and experimental study of dynamic load-carrying capacity for flexible robotic arms in point-to-point motion," Optimal Control Applications and Methods, vol. 38, no. 6, pp. 963-972, 2017.

[39] M. H. Korayem and S. F. Dehkordi, "Dynamic modeling of flexible cooperative mobile manipulator with revolute-prismatic joints for the purpose of moving common object with closed kinematic chain using the recursive Gibbs-appell 
formulation," Mechanism and Machine Theory, vol. 137, pp. 254-279, 2019.

[40] A. Agarwal, S. V. Shah, S. Bandyopadhyay, and S. K. Saha, "Dynamics of serial kinematic chains with large number of degrees-of-freedom," Multibody System Dynamics, vol. 32, no. 3, pp. 273-298, 2014.

[41] M. H. Korayem, V. Azimirad, H. Vatanjou, and A. H. Korayem, "Maximum load determination of nonholonomic mobile manipulator using hierarchical optimal control," Robotica, vol. 30, no. 1, pp. 53-65, 2012.

[42] M. H. Korayem and H. Ghariblu, "Maximum allowable load on wheeled mobile manipulators imposing redundancy constraints," Robotics and Autonomous Systems, vol. 44, no. 2, pp. 151-159, 2003.

[43] M. H. Korayem, A. Heidari, and A. Nikoobin, "Maximum allowable dynamic load of flexible mobile manipulators using finite element approach," International Journal of Advanced Manufacturing Technology, vol. 36, no. 9-10, pp. 1010-1021, 2008.

[44] Y. Xia, J. Wang, and L.-M. Fok, "Grasping-force optimization for multifingered robotic hands using a recurrent neural network," IEEE Transactions on Robotics and Automation, vol. 20, no. 3, pp. 549-554, 2004.

[45] J. Sun, J.-S. Chen, and C.-H. Ko, "Neural networks for solving second-order cone constrained variational inequality problem," Computational Optimization and Applications, vol. 51, no. 2, pp. 623-648, 2012.

[46] X. Miao, J. S. Chen, and C. H. Ko, A Smoothed NR Neural Network for Solving Nonlinear Convex Programs with SecondOrder Cone constraints, Elsevier Science Inc., Amsterdam, Netherlands, 2014.

[47] A. Schmitz, Y. Bansho, K. Noda, K. Iwata, K. Iwata, and K. Sugano, "Tactile object recognition using deep learning and dropout," in Proceedings of the 2014 14th IEEE-RAS international Conference on humanoid robots, pp. 1044-1050, Madrid, Spain, November 2014.

[48] S. Levine, P. Pastor, A. Krizhevsky et al., "Learning hand-eye coordination for robotic grasping with deep learning and large-scale data collection," International Journal of Robotics Research, vol. 37, no. 4-5, pp. 421-436, 2018.

[49] J. Qin, H. Liu, G. Zhang et al., "Grasp stability prediction using tactile information," pp. 498-503, 2017.

[50] Y. Chao, X. Chen, and N. Xiao, "Deep learning-based graspdetection method for a five-fingered industrial robot hand," Iet Computer Vision, vol. 13, no. 1, pp. 61-70, 2019.

[51] C.-H. Ko and J.-S. Chen, "Optimal grasping manipulation for multifingered robots using semismooth Newton method," Mathematical Problems in Engineering, vol. 2013, Article ID 681710, 9 pages, 2013.

[52] C.-H. Ko, J.-S. Chen, and C.-Y. Yang, "Recurrent neural networks for solving second-order cone programs," Neurocomputing, vol. 74, no. 17, pp. 3646-3653, 2011.

[53] J.-T. Li, Grasp Planning for Robotic Multi-Fingered hands, Beihang University, Beijing, China, 2004.

[54] R. S. Feijóo, J. Cornellà, and M. R. Garzón, "Grasp quality measures," Recercat Home, vol. 38, no. 1, pp. 65-88, 2006.

[55] K. L. Johnson, Contact Mechanics, Cambridge University Press, Cambridge, UK, 1985.

[56] H. M. Lankarani and P. E. Nikravesh, "A contact force model with hysteresis damping for impact analysis of multibody systems," Journal of Mechanical Design, vol. 112, no. 3, pp. 369-376, 1990.

[57] F. Pfeiffer and G. Christoph, Multibody Dynamics with Unilateral Contacts, Wiley VCH, New York, NY, USA, 1996.
[58] K. Ye, L. Li, and H. Zhu, "A note on the Hertz contact model with nonlinear damping for pounding simulation," Earthquake Engineering \& Structural Dynamics, vol. 38, no. 9, pp. 1135-1142, 2010.

[59] P. Jia, W. 1. Li, G. Wang, and S. Y. Li, "Optimal grasp planning for a dexterous robotic hand using the volume of a generalized force ellipsoid during accepted flattening," International Journal of Advanced Robotic Systems, vol. 14, no. 1, Article ID $172988141668713,2017$.

[60] P. Jia, L. Q. Wang, W. L. Li et al., "Research on contact constraints linearization of dexterous hand and grasping forces optimization," in Proceedings of the International Conference on Mechanics and Mechanical Engineering, pp. 97-107, Chengdu, China, 2016.

[61] Y.-R. Zhang, J.-T. Li, and J.-F. Li, Robot Dexterous Hand: Modeling, Planning and Simulation, China Machine Press, Beijing, China, 2007. 


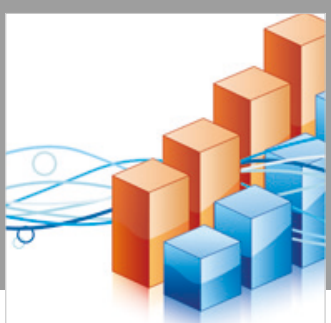

Advances in

Operations Research

\section{-n-m}

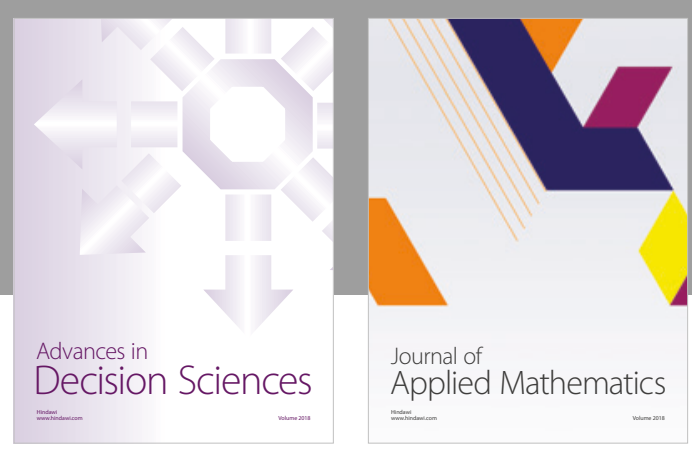

Journal of

Applied Mathematics


Submit your manuscripts at

www.hindawi.com

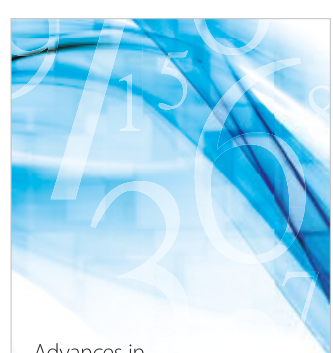

Advances in
Numerical Analysis
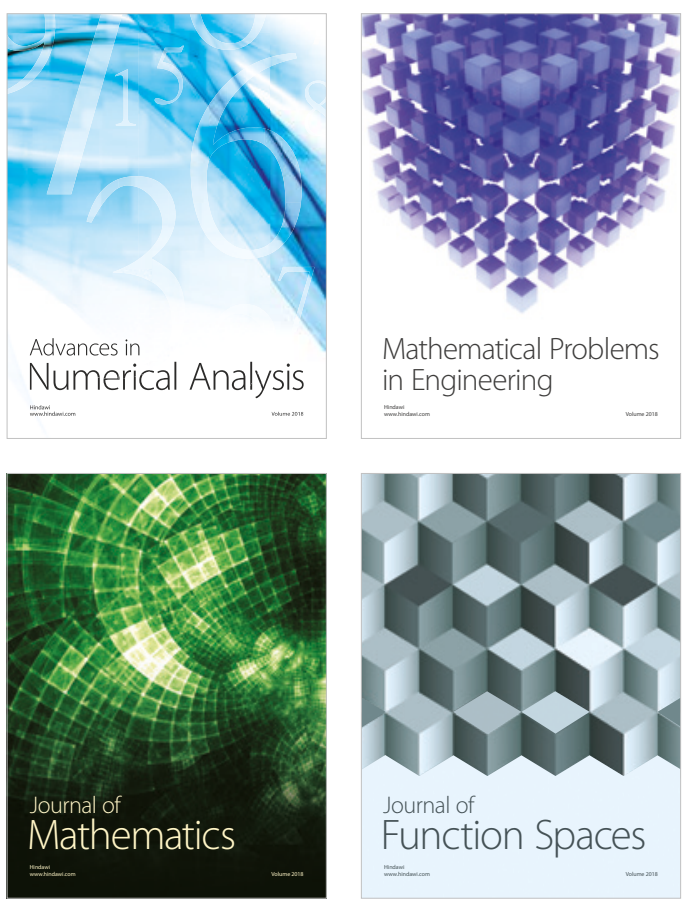

Mathematical Problems in Engineering

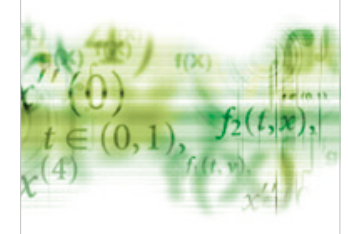

International Journal of

Differential Equations

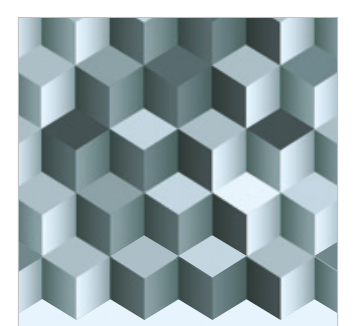

Journal of

Function Spaces
The Scientific

World Journal

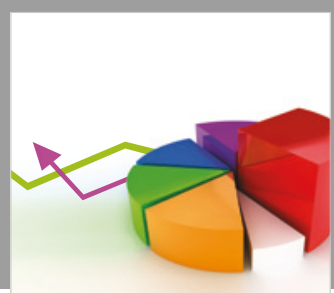

Journal of

Probability and Statistics
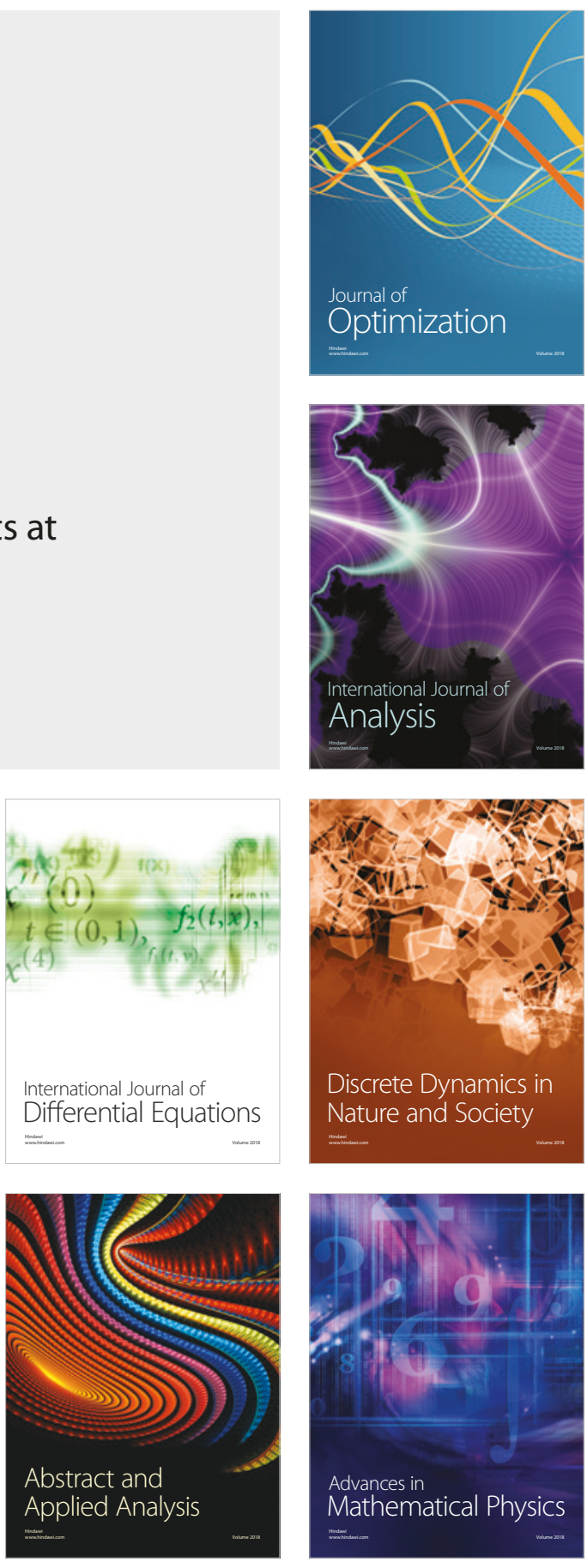